\title{
Search for electroweak production of a vector-like quark decaying to a top quark and a Higgs boson using boosted topologies in fully hadronic final states
}

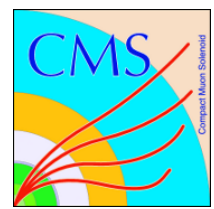

\section{The CMS collaboration}

E-mail: cms-publication-committee-chair@cern.ch

ABSTRACT: A search is performed for electroweak production of a vector-like top quark partner $\mathrm{T}$ of charge $2 / 3$ in association with a standard model top or bottom quark, using $2.3 \mathrm{fb}^{-1}$ of proton-proton collision data at $\sqrt{s}=13 \mathrm{TeV}$ collected by the CMS experiment at the CERN LHC. The search targets $\mathrm{T}$ quarks decaying to a top quark and a Higgs boson in fully hadronic final states. For a $\mathrm{T}$ quark with mass above $1 \mathrm{TeV}$ the daughter top quark and Higgs boson are highly Lorentz-boosted and can each appear as a single hadronic jet. Jet substructure and $b$ tagging techniques are used to identify the top quark and Higgs boson jets, and to suppress the standard model backgrounds. An excess of events is searched for in the $\mathrm{T}$ quark candidate mass distribution in the data, which is found to be consistent with the expected backgrounds. Upper limits at $95 \%$ confidence level are set on the product of the single $\mathrm{T}$ quark production cross sections and the branching fraction $\mathcal{B}(\mathrm{T} \rightarrow \mathrm{tH})$, and these vary between 0.31 and $0.93 \mathrm{pb}$ for $\mathrm{T}$ quark masses in the range $1000-1800 \mathrm{GeV}$. This is the first search for single electroweak production of a vector-like $\mathrm{T}$ quark in fully hadronic final states.

Keywords: Beyond Standard Model, Hadron-Hadron scattering (experiments), Top physics

ARXIV EPRINT: 1612.05336 


\section{Contents}

1 Introduction 1

2 Signal and background modeling 3

3 The CMS detector and event reconstruction 4

4 Event selection $\quad 5$

$\begin{array}{lll}5 & \text { Background estimation } & 7\end{array}$

6 Systematic uncertainties $\quad 9$

$\begin{array}{lll}7 & \text { Results } & 10\end{array}$

$\begin{array}{lll}8 & \text { Summary } & 11\end{array}$

$\begin{array}{ll}\text { The CMS collaboration } & 17\end{array}$

\section{Introduction}

We report on a search for a vector-like top quark partner $(\mathrm{T})$ of charge $2 / 3$. The $\mathrm{T}$ quark appears in many extensions of the standard model (SM) and usually mixes with the SM top quark [1-6]. Vector-like quarks (VLQs) like the T could have a role in regularizing the SM Higgs boson $(\mathrm{H})$ mass, thus offering a solution to the hierarchy problem $[7,8]$. The production of VLQs can be in pairs via the strong interaction, or singly in association with the SM top or bottom quarks via the electroweak interaction. The electroweak couplings of the T quarks to the SM third-generation quarks are highly model dependent. These couplings determine the rates of the single $\mathrm{T}$ quark production modes, shown in figure 1 . The expected decay channels of a $\mathrm{T}$ quark coupling to the SM top or bottom quarks are $\mathrm{T} \rightarrow \mathrm{bW}, \mathrm{T} \rightarrow \mathrm{tZ}$, and $\mathrm{T} \rightarrow \mathrm{tH}[9]$. Probing such processes could shed light on the mixing of VLQs with the SM third-generation quarks.

The VLQs are non-chiral particles, i.e., their left-handed (LH) and right-handed (RH) components are part of the same multiplet under a weak isospin symmetry transformation. As a consequence, their masses are not restricted by their Yukawa couplings to the Higgs field; hence these particles are not ruled out by constraints from measurements of the production and decay rates of the Higgs boson [10].

Searches for pair-production of T quarks have been conducted by the ATLAS and CMS collaborations at the CERN LHC using proton-proton (pp) collision data at $\sqrt{s}=$ $8 \mathrm{TeV}$, and limits placed on the mass between 720 and $950 \mathrm{GeV}$, depending on the decay mode $[11,12]$. A search for single production of $\mathrm{T}$ quarks decaying to $\mathrm{Wb}$ was conducted 

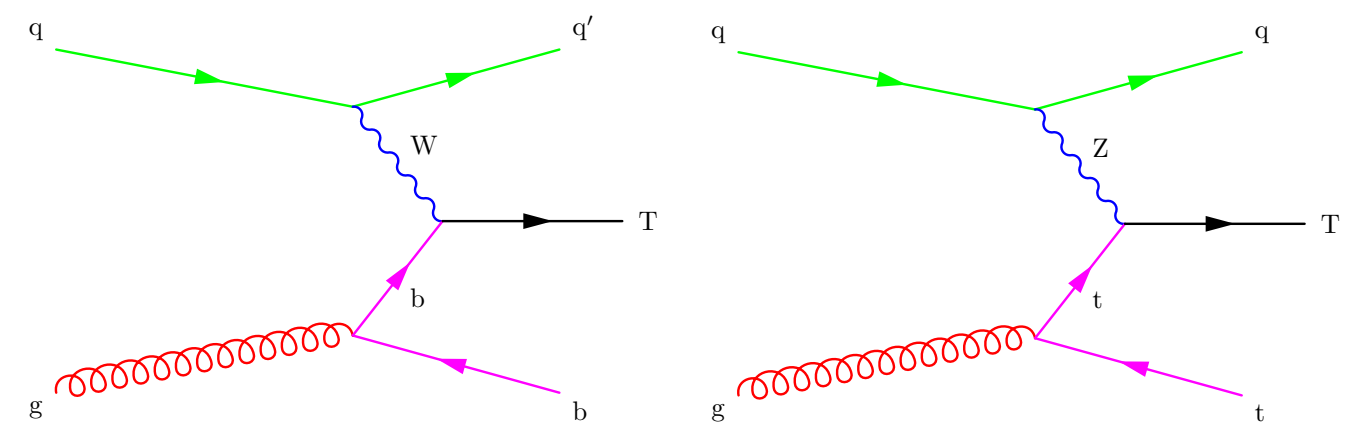

Figure 1. Example production diagrams for the processes pp $\rightarrow$ Tbq via the charged current (left) and $\mathrm{pp} \rightarrow$ Ttq via the neutral current (right).

by the ATLAS collaboration using pp collision data at $\sqrt{s}=8 \mathrm{TeV}$, and a limit on the $\mathrm{T}$ quark mass was set at $950 \mathrm{GeV}$ [13]. For high VLQ masses, the pair-production cross section rapidly decreases as the phase space for producing two massive particles is limited. Above the $\mathrm{TeV}$ range, single production via the electroweak process is expected to dominate over pair production [14], and is thus the focus of this search.

In this Letter we present a search for a singly produced $\mathrm{T}$ quark using pp collision data collected at $\sqrt{s}=13 \mathrm{TeV}$ with the CMS experiment in 2015, and corresponding to an integrated luminosity of $2.3 \mathrm{fb}^{-1}$. The production processes considered are $\mathrm{pp} \rightarrow \mathrm{Tbq}$ and $\mathrm{pp} \rightarrow \mathrm{Ttq}$, as shown in figure 1 . We consider the decay mode $\mathrm{T} \rightarrow \mathrm{tH}$ with the top quark decaying fully hadronically $\left(\mathrm{t} \rightarrow \mathrm{bW} \rightarrow \mathrm{bq} \overline{\mathrm{q}^{\prime}}\right)$ and the SM Higgs boson decaying to $\mathrm{b} \overline{\mathrm{b}}$. For a SM Higgs boson with a mass close to $125 \mathrm{GeV}$ [15], the decay branching fraction $\mathcal{B}(\mathrm{H} \rightarrow \mathrm{b} \overline{\mathrm{b}})=58 \%$ [16]. Recently, a companion CMS analysis has searched for a singly-produced $\mathrm{T}$ quark with $\mathrm{T} \rightarrow \mathrm{tH}$ using this $13 \mathrm{TeV}$ data set in a leptonic final state [17], and set limits on the product of the $\mathrm{T}$ quark cross section and the branching fraction $\mathcal{B}(\mathrm{T} \rightarrow \mathrm{tH})$ in the mass range $1000-1800 \mathrm{GeV}$.

The pp $\rightarrow$ Tbq with $\mathrm{T} \rightarrow \mathrm{tH}$ channel contains seven outgoing partons while the $\mathrm{pp} \rightarrow \mathrm{Ttq}$ with $\mathrm{T} \rightarrow \mathrm{tH}$ channel contains nine, from the hard scattering process. These partons subsequently hadronize to produce jets. For $\mathrm{T}$ quarks with a mass above $1 \mathrm{TeV}$, the decay products of the top quark and the Higgs boson are highly Lorentz-boosted and collimated, producing two hadronic jets. The accompanying jets are softer. Thus, the signature of a massive $\mathrm{T}$ quark would be the presence of highly boosted jets with masses corresponding to those of the top quark and the Higgs boson, and an overall large hadronic activity in the event. The $\mathrm{T}$ quark candidates are reconstructed using the top quark and Higgs boson jets. In the T quark candidate mass distribution, a localized excess of events above the SM background is expected in the presence of a signal.

This is the first search for single electroweak production of a vector-like $\mathrm{T}$ quark in fully hadronic final states. Jet substructure and b tagging techniques are employed to identify the highly Lorentz-boosted top quark and Higgs boson arising from the decay of a $\mathrm{TeV}$ scale resonance. The search in these final states exploits the ability of jet substructure techniques to reconstruct hadronically decaying SM particles in a challenging fully hadronic environment. 


\section{Signal and background modeling}

The single $\mathrm{T}$ quark production cross section and the branching fraction $\mathcal{B}(\mathrm{T} \rightarrow \mathrm{tH})$ are highly model dependent. The Simplest Simplified Model (SSM) framework [18] is used to model the signal events. In this framework, the coupling factors $c_{\mathrm{L} / \mathrm{R}}^{\mathrm{bW}}$ and $c_{\mathrm{L} / \mathrm{R}}^{\mathrm{tZ}}$ determine the strengths of the charged and neutral current interactions, as shown in figure 1 left and right, respectively, up to a factor of the electroweak coupling constant $g_{\mathrm{W}}$. Signal events for the processes pp $\rightarrow$ Tbq and pp $\rightarrow$ Ttq are generated for $\mathrm{LH}$ or $\mathrm{RH}$ interactions, with each of the corresponding LH or RH coupling factors set to unity, while the other is set to zero. Events are generated using the tree-level Monte Carlo (MC) event generator MadGraph 5.1.3.30 [19] for T quark masses from 1000 to $1800 \mathrm{GeV}$, in steps of $100 \mathrm{GeV}$. The signal widths are set to $10 \mathrm{GeV}$ for all masses. The NNPDF3.0 [20] parton distribution function (PDF) set is used.

The main SM background processes are $t \bar{t}+$ jets and multijet production through the strong interaction. A smaller contribution comes from $\mathrm{W}+$ jets events. Events with a single top quark and a $\mathrm{W}$ boson (tW) are found to make a negligible contribution to the overall background composition. The $\mathrm{t} \overline{\mathrm{t}}+\mathrm{jets}, \mathrm{W}+\mathrm{jets}$, and $\mathrm{tW}$ background events are estimated using MC simulations. As it is difficult to accurately simulate multijet production, the contributions from these processes are estimated from data. All other SM processes have a negligible contribution to the background.

A next-to-next-to-leading order (NNLO) $t \bar{t}+$ jets production cross section of $832_{-51}^{+46} \mathrm{pb}$ [21], corresponding to a top quark mass of $172.5 \mathrm{GeV}$, is used to estimate the $\mathrm{t} \overline{\mathrm{t}}+\mathrm{jets}$ rate. The NNLO tW production cross section is taken as $71.7 \pm 3.8 \mathrm{pb}[22,23]$. The $\mathrm{t} \overline{\mathrm{t}}+\mathrm{jets}$ events and the tW events are simulated using PowHEg v2 [24-27] with inclusive top quark decays.

The $\mathrm{W}+$ jets events cross section is estimated to be $95.1 \pm 3.6 \mathrm{pb}$, calculated at NNLO with FEwZ 3.1 [28]. This cross section is calculated in the region of phase space where the sum of the parton transverse momenta $p_{\mathrm{T}}$ is greater than $600 \mathrm{GeV}$, in keeping with the signal event topology requiring highly boosted jets. The $\mathrm{W}+$ jets production sample, simulated with MADGRAPH 5.1.3.30, is restricted to this phase space and to events with hadronic $\mathrm{W}$ boson decays. The uncertainties in the cross sections of these processes derive from the uncertainties in the PDFs, the quantum chromodynamics (QCD) factorization and renormalization scales, and the strong coupling constant.

The multijet samples are generated using MADGRAPH 5.1.3.30 with up to four partons included in the matrix element calculation, and are used only to optimize the event selection and validate the background estimation procedure.

The samples generated using the MADGRAPH 5.1.3.30 or POWHEG v2 programs are interfaced with PYTHIA 8.212 [29] for showering and hadronization, using the underlying event tune CUETP8M1 [30], and with the MLM matching scheme [31] to match the additional partons from the hard process with those simulated using the parton shower algorithm. In all simulations, the mass of the Higgs boson is set to $125 \mathrm{GeV}$, while the top quark mass is set to $172.5 \mathrm{GeV}$.

Additional pp interactions (pileup) in concurrence with the hard interaction are simulated by overlaying low $p_{\mathrm{T}} \mathrm{QCD}$ interactions, using the PYTHIA 8.212 MC generator and 
a total inelastic pp cross section of $69 \mathrm{mb}$ [32]. The distribution of the number of pileup events in the simulated samples is reweighted to match the distribution observed in the data. The generated signal events are processed using a GEANT4-based [33, 34] simulation of the CMS detector.

\section{The CMS detector and event reconstruction}

The CMS detector, its coordinate system, and its kinematic variables are detailed in ref. [35]. The detector consists of a superconducting solenoid of $6 \mathrm{~m}$ internal diameter at its core, providing a magnetic field of $3.8 \mathrm{~T}$. Within the field volume are housed a silicon pixel and strip tracker, a lead tungstate crystal electromagnetic calorimeter (ECAL), and a brass and scintillator hadron calorimeter (HCAL), each of which is divided into a barrel and two endcap sections. The tracker extends from -2.5 to +2.5 in pseudorapidity $\eta$ while the ECAL and the HCAL extend up to $|\eta|=3$. Extensive forward calorimetry, up to $|\eta|=5$, complements the coverage provided by the barrel and endcap detectors. Muons are measured in gas-ionization detectors embedded in the steel flux-return yoke outside the solenoid, covering a region of $|\eta|<2$.4.

Events are selected using a two-stage trigger system, requiring the presence of hadronic jets in the detector. The level-1 trigger selects events with jets, reconstructed from energy deposits in the ECAL and the HCAL, for further processing by the high-level trigger (HLT). The HLT reconstructs jets with $p_{\mathrm{T}}>40 \mathrm{GeV}$ and $|\eta|<3$ that are clustered using the particle flow $(\mathrm{PF})$ algorithm $[36,37]$ described below. The scalar sum of the jet $p_{\mathrm{T}}$ $\left(H_{\mathrm{T}}\right)$ is required to be greater than $800 \mathrm{GeV}$ for the event to be selected by the HLT for further processing.

Charged particle tracks are used to reconstruct the interaction vertices. The vertex with the highest sum of the $p_{\mathrm{T}}^{2}$ of clusters of associated tracks is chosen as the primary vertex. The PF event reconstruction algorithm reconstructs and identifies stable particles in the detector using an optimized combination of information from all subdetectors. The $\mathrm{PF}$ candidates are used to reconstruct jets using the anti- $k_{\mathrm{T}}$ algorithm [38] implemented using the FASTJET package $[39,40]$. Charged particles not originating from the primary vertex are omitted in the jet clustering. The jet momentum is the vector sum of the momenta of all particles clustered in the jet.

The jet energy scale is determined from a detailed simulation of the CMS detector. The estimated pileup contribution to the jet energy is subtracted using an event-by-event jet area based correction [41, 42]. Further corrections are applied to account for the detector response to hadrons as a function of the jet $p_{\mathrm{T}}$ and $\eta$. Additional corrections are then applied to the data to account for any remaining differences with the simulations in the jet energy measurement. From simulations, the average jet momentum is found to be within $5 \%$ of the true momentum over the whole range of detector acceptance. The jet energy resolution varies from $15-20 \%$ at $30 \mathrm{GeV}$ to $5 \%$ at $1 \mathrm{TeV}$ [43].

Two non-exclusive jet collections are reconstructed, one by clustering the PF candidates using the anti- $k_{\mathrm{T}}$ distance parameter, in the $\eta-\phi$ plane, of 0.4 (AK4 jets), and the other using a distance parameter of 0.8 (AK8 jets). The former is used to calculate the 
$H_{\mathrm{T}}$, while the latter is used to reconstruct Lorentz-boosted top quark and Higgs boson jets. Jets are required to pass a standard set of quality criteria to reject detector and electronics noise misidentified as jets [44].

\section{Event selection}

Events passing the jet-based trigger are further required to have at least four AK4 jets with $p_{\mathrm{T}}>30 \mathrm{GeV}$ and $|\eta|<5$, and at least one AK8 jet with $p_{\mathrm{T}}>300 \mathrm{GeV}$ and $|\eta|<2.4$. We further require that the $H_{\mathrm{T}}$ of such an event, where the sum of the $p_{\mathrm{T}}$ is taken of all selected AK4 jets, is greater than $1100 \mathrm{GeV}$. These together constitute the preselection criteria for further processing of an event. The trigger efficiency of events passing the preselection criteria is found to be $100 \%$ with negligible uncertainty.

Jet grooming techniques [45] are applied to AK8 jets to identify hadronic decays of Lorentz-boosted massive particles like $\mathrm{H} \rightarrow \mathrm{b} \overline{\mathrm{b}}$ or $\mathrm{t} \rightarrow \mathrm{bq} \overline{\mathrm{q}^{\prime}}$. The pruning [46] and soft-drop [47, 48] grooming algorithms are employed to remove soft contributions to the jet energy from the underlying event and pileup, and to reveal subjets coming from the hadronization of the hard partons arising from the massive particle decay. The mass of the jet is thus closer to that of the massive parent particle after grooming, and the subjets can be associated with its decay products. The groomed AK8 jets are required to pass further selection criteria to be identified either as Higgs boson-tagged (H-tagged), or top quarktagged (t-tagged) jets. Values chosen for the selection parameters associated with the jet pruning and soft-drop algorithms, as well as with the N-subjettiness algorithm described below, are based on detailed studies of their performance in a sample of semileptonic $t \bar{t}+$ jets events with t-tagged or $\mathrm{W}$ boson-tagged jets, as described in refs. [49, 50].

The pruning parameters used are $z_{\text {cut }}=0.1$ and $D_{\text {cut }}=0.5$, while the soft-drop parameters are set to $z_{\text {cut }}=0.1$ and $\beta=0$. Both the pruning and the soft-drop algorithms are applied to the same set of AK8 jets, yielding the pruned and soft-drop masses, respectively. The H-tagged jets require a pruned mass between $105-135 \mathrm{GeV}$. The t-tagged jets require a soft-drop mass within $110-210 \mathrm{GeV}$. The soft-drop subjets are further used for $\mathrm{b}$ tagging, for both the H-tagged and t-tagged jets. The combination of pruned mass for $\mathrm{H}$ tagging [51] and soft-drop mass for t tagging [52] was found to give the best rejection of pileup events and other backgrounds.

Besides the pruning and the soft-drop algorithms, the N-subjettiness algorithm [53], based on the computation of the inclusive jet shape variables $\tau_{N}$, is used. These variables quantify "lobes" of energy flow inside a jet [53]. A jet compatible with two substructures would have values of the ratio $\tau_{2} / \tau_{1}$ much less than unity, as in a boosted $\mathrm{H} \rightarrow \mathrm{b} \overline{\mathrm{b}}$ decay. Likewise, a jet from a boosted $\mathrm{t} \rightarrow \mathrm{bq \overline {q^{ \prime } }}$ decay, with three substructures, would have the value of $\tau_{3} / \tau_{2}$ much less than one. In contrast, jets with no substructure would exhibit larger values for both $\tau_{2} / \tau_{1}$ and $\tau_{3} / \tau_{2}$. Thus these variables provide good discrimination against multijet backgrounds. The requirements on the ratios $\tau_{2} / \tau_{1}<0.6$ and $\tau_{3} / \tau_{2}<0.54$ are used for $\mathrm{H}$ and $\mathrm{t}$ tagging, respectively.

The soft-drop subjets are b-tagged to further suppress backgrounds. The combined secondary vertex b tagging algorithm (CSVv2) identifies subjets containing B hadrons 
using a combination of track and secondary vertex related variables [54]. For $\mathrm{H}$ tagging, the CSVv2 discriminator threshold is chosen to give a mistag rate of $10 \%$ for subjets from light flavored quarks and gluons, and a signal efficiency of 40-70\%, depending on the subjet $p_{\mathrm{T}}[55]$. Both of the subjets are required to pass the $\mathrm{b}$ tagging requirement. Boosted jets with both subjets failing the $\mathrm{b}$ tagging criteria but otherwise satisfying the $\mathrm{H}$ tagging criteria ("anti-H-tagged") are used to define a control region for background estimation. For $\mathrm{t}$ tagging, one subjet is required to have a CSVv2 discriminator value that exceeds a more stringent threshold, to give an overall mistag rate of about $0.1 \%$ [50].

Jet energy scale corrections are applied to the H-tagged jet mass to obtain a better agreement with the Higgs boson mass. The H-tagged jet mass resolution in the simulations is degraded to match the observed $W$ jet mass resolution in the data in a sample of $t \bar{t}+$ jets events with boosted hadronically decaying $\mathrm{W}$ bosons. The $\mathrm{W}$ jets are tagged in the same way as $\mathrm{H}$-tagged jets, except that the pruned mass is required to be within the range $65-$ $105 \mathrm{GeV}$, and the subjets are not b-tagged. The $\mathrm{W}$ jets are also used to obtain the ratio of the N-subjettiness selection efficiencies between the data and the simulations, which is applied to the simulated $\mathrm{H}$ jets as a scale factor. A simulation-based correction factor is applied to account for the difference in the jet shower profile of $\mathrm{W} \rightarrow \mathrm{q} \overline{\mathrm{q}^{\prime}}$ and $\mathrm{H} \rightarrow \mathrm{b} \overline{\mathrm{b}}$ decays. The $b$ tagging efficiency scale factors, measured on a sample of jets with subjets required to contain a muon to enrich them in B hadron flavor [55], are likewise applied. The t jet tagging efficiency scale factor is obtained from boosted hadronically decaying top quarks where the decay products of the daughter $\mathrm{W}$ boson and the $\mathrm{b}$ quarks are merged and clustered as one AK8 jet $[49,50]$.

It is observed that the MC simulations of the background do not model well the jet $p_{\mathrm{T}}$ and $H_{\mathrm{T}}$ distributions after the preselection [17]. The data/MC ratio of the $H_{\mathrm{T}}$ distribution is described within statistical uncertainties by a 2-parameter linear fit with a significant negative slope parameter. The $H_{\mathrm{T}}$ distributions of background components obtained from MC simulations are reweighted using the results of this fit. Cross-checks are performed in different control regions confirming the validity of this correction factor. The correction factor is applied to the predicted background, with a small impact on the background $\mathrm{T}$ quark candidate mass distribution. The $H_{\mathrm{T}}$ reweighting has a negligible effect on the signal and is considered only as a systematic uncertainty.

The H-tagged and t-tagged jets are required to have $p_{\mathrm{T}}$ values greater than 300 and $400 \mathrm{GeV}$, respectively. An AK8 jet that is simultaneously H-tagged and t-tagged is assigned to the latter category, although this occurs in less than $1 \%$ of the events. Furthermore, the $\mathrm{H}$-tagged and t-tagged jets must have a separation in the $\eta-\phi$ plane, $\Delta R(\mathrm{H}, \mathrm{t})>$ 2.0. These selection criteria define the signal region. The highest $p_{\mathrm{T}} \mathrm{H}$-tagged and $\mathrm{t}-$ tagged jets satisfying the above requirements in each signal event are paired to form the $\mathrm{T}$ quark candidate, where the $\mathrm{T}$ quark mass, $M(\mathrm{~T})$, is taken to be the invariant mass of the dijet system.

The search is performed by looking for a localized excess in the $M(\mathrm{~T})$ distribution above the SM background. The simulated reconstructed $M(\mathrm{~T})$ distributions for a few representative masses are shown in figure 2. The estimated mass resolution of the $\mathrm{T}$ quark candidates is about $5 \%$ for all simulated T quark masses. Table 1 gives some representative 


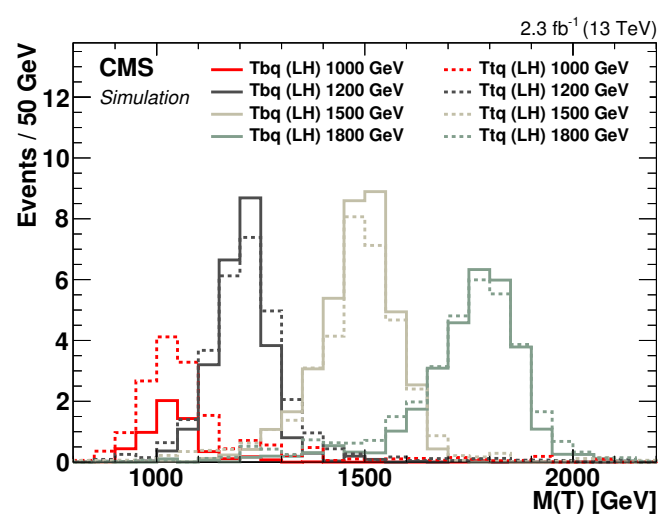

Figure 2. The simulated $M(\mathrm{~T})$ distributions for the $\mathrm{pp} \rightarrow$ Tbq and the $\mathrm{pp} \rightarrow$ Ttq production modes with left-handed coupling for the T quark masses 1000, 1200, 1500, and $1800 \mathrm{GeV}$, after all selection criteria have been applied. The values of the product of the signal cross sections and the branching fraction $\mathcal{B}(\mathrm{T} \rightarrow \mathrm{tH})$ are taken to be $1 \mathrm{pb}$.

\begin{tabular}{|l|ccc|ccc|}
\hline \multirow{3}{*}{ Mass $(\mathrm{GeV})$} & \multicolumn{6}{|c|}{ Efficiency $(\%)$} \\
\cline { 2 - 7 } & \multicolumn{2}{|c|}{$\mathrm{pp} \rightarrow$ Tbq, $\mathrm{T} \rightarrow \mathrm{tH}(\mathrm{LH})$} & \multicolumn{3}{c|}{$\mathrm{pp} \rightarrow$ Ttq, $\rightarrow \mathrm{TH}(\mathrm{LH})$} \\
\cline { 2 - 7 } & Preselection & $\mathrm{H}$ tag & $\mathrm{t}$ tag & Preselection & $\mathrm{H}$ tag & $\mathrm{t}$ tag \\
\hline 1000 & 15.7 & 3.1 & 0.35 & 51.5 & 9.1 & 0.8 \\
1100 & 24.9 & 5.7 & 0.8 & 61.1 & 11.4 & 1.1 \\
1200 & 36.4 & 8.7 & 1.1 & 69.1 & 12.8 & 1.3 \\
1300 & 44.6 & 10.6 & 1.4 & 74.8 & 13.8 & 1.4 \\
1400 & 52.0 & 11.8 & 1.6 & 79.4 & 14.9 & 1.6 \\
1500 & 57.1 & 12.6 & 1.7 & 82.1 & 15.1 & 1.6 \\
1700 & 63.3 & 12.9 & 1.5 & 86.3 & 15.8 & 1.7 \\
1800 & 65.6 & 13.2 & 1.4 & 87.9 & 15.5 & 1.5 \\
\hline
\end{tabular}

Table 1. The signal efficiencies for successive event selections for the $\mathrm{pp} \rightarrow \mathrm{Tbq}$ and $\mathrm{pp} \rightarrow \mathrm{Ttq}$ models with left-handed couplings. The preselection criteria are more efficient for the $\mathrm{pp} \rightarrow \mathrm{Ttq}$ process compared to the pp $\rightarrow$ Tbq process owing to the larger number of jets, and hence a higher $H_{\mathrm{T}}$ per event, in the former. This is more pronounced for low $\mathrm{T}$ quark mass samples, where the $H_{\mathrm{T}}$ is close to the trigger threshold of $800 \mathrm{GeV}$.

signal efficiencies for different $\mathrm{T}$ quark masses, for the $\mathrm{pp} \rightarrow \mathrm{Tbq}$ and $\mathrm{pp} \rightarrow$ Ttq processes with LH couplings. The effective integrated luminosities of the simulated signal samples are much larger than the integrated luminosity corresponding to the data; hence the statistical uncertainties in the efficiencies are negligible. The efficiencies for the $\mathrm{RH}$ couplings are very similar to those for the LH couplings of the corresponding models.

\section{Background estimation}

The main backgrounds in the signal region are $t \bar{t}+$ jets, multijets, and, to a lesser extent, $\mathrm{W}+$ jets. The tW background is negligible, with none of the simulated events passing the full event selection, from a sample whose corresponding integrated luminosity is much 


\begin{tabular}{|lcccc|}
\hline & & $\mathrm{A}$ & $\mathrm{B}$ & $\mathrm{C}$ \\
\hline Data & & 94126 & 207 & 7251 \\
\hline \multirow{3}{*}{ Non-multijet bkg. } & $\mathrm{t} \overline{\mathrm{t}}+$ jets & $812 \pm 6$ & $53.9 \pm 1.5$ & $366 \pm 4$ \\
& W+jets & $1258 \pm 25$ & $6.0 \pm 1.8$ & $109 \pm 8$ \\
& $\mathrm{tW}$ & $27 \pm 2$ & $0.5 \pm 0.2$ & $12.7 \pm 1.1$ \\
\hline Data - non-multijet bkg. & & $92029 \pm 26$ & $146.6 \pm 2.4$ & $6763 \pm 9$ \\
\hline
\end{tabular}

Table 2. Numbers of events for the control regions $A, B$, and $C$ in the non-multijet backgrounds, and the differences between numbers of events in the data and these backgrounds. These differences are attributed to the multijet background. The uncertainties are statistical only.

larger than that of the data sample. All backgrounds except multijets are estimated using simulations.

The multijet background is estimated from the data by using four selection regions $A, B, C$, and $D$. Events in region $A$ are required to have at least one anti-H-tagged jet and no $\mathrm{H}$-tagged or t-tagged jets, while those in region $B$ are required to have at least one anti-H-tagged jet and at least one t-tagged jet, and zero $\mathrm{H}$-tagged jets. Events in region $C$ should have at least one $\mathrm{H}$-tagged jet and zero t-tagged jets. Region $D$ is the signal region and contains events with at least one $\mathrm{H}$-tagged and one t-tagged jet, as defined in the previous section. The $t \overline{\mathrm{t}}+\mathrm{jets}, \mathrm{W}+\mathrm{jets}$, and the $\mathrm{tW}$ backgrounds all contribute to the $A, B$, and $C$ regions.

The independence of the two variables that span the $A, B, C$, and $D$ regions, i.e., the $\mathrm{H}$ tagging or anti-H-tagging, and the t tagging criteria, was validated using simulations. Since the two variables are uncorrelated, the number of events $N_{A, B, C, D}$ for the corresponding regions should follow the relation $N_{A} / N_{B}=N_{C} / N_{D}$. Thus, the number of background events in the signal region $D$ would be determined by the number of events in the three control regions: $N_{D}=N_{B} N_{C} / N_{A}$.

The $A B C D$ method is also used to obtain the background $M(\mathrm{~T})$ distribution for the signal region. The anti-H-tagged and t-tagged jets are paired to reconstruct the $M(\mathrm{~T})$ shape in the control region $B$. When multiplied by the ratio $N_{C} / N_{A}$, this gives the background $M(\mathrm{~T})$ shape in the signal region. A validation of the procedure is performed using simulations. The compatibility of the shapes in the $B$ and $D$ regions are verified using simulated QCD multijet samples. Moreover, the shapes of the data and simulation distributions in region $B$ are found to be consistent, and thus the $A B C D$ method is also expected to correctly predict the multijet background in the signal region $D$ from the data in regions $A, B$, and $C$.

Since only the multijet background is estimated using the $A B C D$ method, the simulated $\mathrm{t} \overline{\mathrm{t}}+\mathrm{jets}, \mathrm{W}+$ jets, and $\mathrm{tW}$ backgrounds are subtracted from the data in each of the $A$, $B$, and $C$ regions to obtain the predicted multijet background in data for that region. The resulting numbers of events in the control regions are given in table 2. The ratio $N_{C} / N_{A}$ is found to be $(7.4 \pm 0.1) \times 10^{-2}$.

The total estimated background from all sources is given in table 3, along with the number of observed events in the data. Since the backgrounds estimated using MC simulations are subtracted from the data in the control regions to estimate the multijets 


\begin{tabular}{|lc|}
\hline Process & \multicolumn{1}{c|}{ Events } \\
\hline Multijets (using data) & $10.8 \pm 5.5$ \\
$\mathrm{t} \overline{\mathrm{t}}+$ jets (using MC) & $24.3 \pm 8.1$ \\
$\mathrm{~W}+$ jets (using MC) & $0.6 \pm 0.6$ \\
\hline Total background & $35.7 \pm 5.6$ \\
\hline Observed events & 30 \\
\hline
\end{tabular}

Table 3. Estimated background and the number of observed events in the signal region after all selection criteria. The combined statistical and systematic uncertainty is shown.
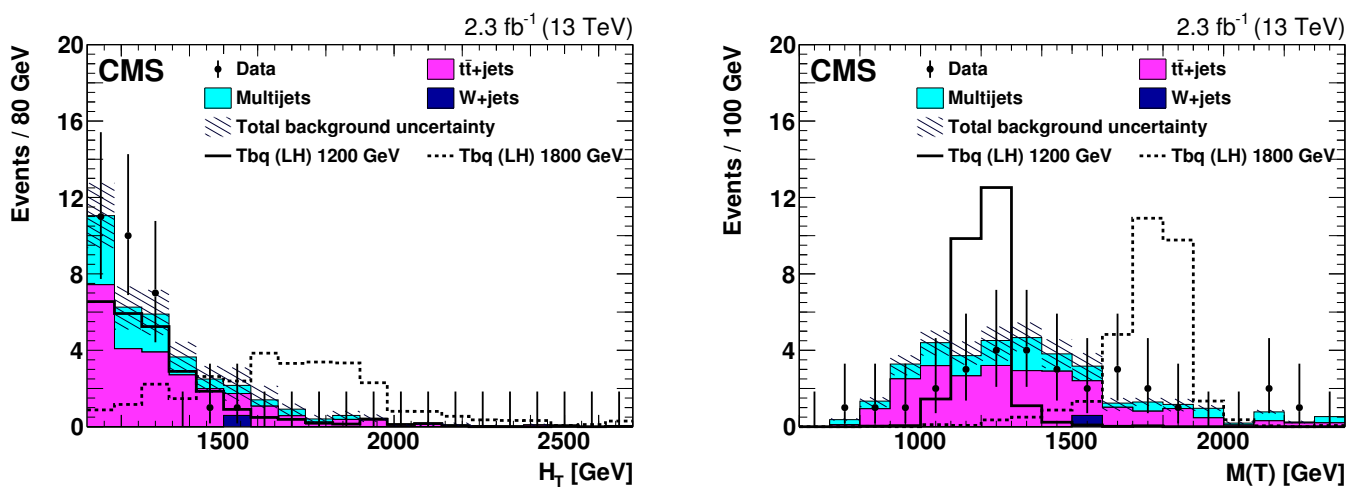

Figure 3. The $H_{\mathrm{T}}$ (left) and $M(\mathrm{~T})$ (right) distributions after full event selection. The black markers with error bars are the data. The various background components are shown as filled histograms, and are estimated using simulations $(t \bar{t}+$ jets and $\mathrm{W}+$ jets) and the data (non- $-\overline{\mathrm{t}}+\mathrm{jets}$ and non-W+jets multijets component). The simulated $\mathrm{T}$ quark signal distributions for two $\mathrm{T}$ quark masses are also shown. The values of the product of the signal cross sections and the branching fraction $\mathcal{B}(\mathrm{T} \rightarrow \mathrm{tH})$ are taken to be $1 \mathrm{pb}$.

component of the background, the associated systematic uncertainties are anticorrelated between the simulated backgrounds and the multijets background. Hence the uncertainty in the total background is less than what one would obtain if the uncertainties in the individual backgrounds were added in quadrature. The $H_{\mathrm{T}}$ and $M(\mathrm{~T})$ distributions in the data, estimated backgrounds, and the simulated signal are shown in figure 3. The overall level of agreement between the observed number of events and the background from the SM processes is within the estimated uncertainties (discussed in section 6).

\section{Systematic uncertainties}

There are two types of systematic uncertainties in the signal and background predictions: those that affect only the total rate, and those that affect the rate and the $M(\mathrm{~T})$ distribution. Among the former are the integrated luminosity uncertainty of $2.7 \%$ [56], the pileup reweighting uncertainty of $5 \%$ in the total inelastic pp collision cross section, the cross section uncertainties in the simulated background predictions, and the uncertainties of 1-3\% from the choice of the PDF set, estimated using the PDF4LHC procedure [57]. 
The scale factor uncertainty due to the $\mathrm{N}$-subjettiness selection for $\mathrm{H}$ tagging is $12.5 \%$, and affects only the total event rate.

The jet energy and mass correction and resolution uncertainties affect the shapes of the $M(\mathrm{~T})$ distributions for both the simulated signal and background processes. The jet energy scale uncertainty is $1-2 \%$ and the jet energy resolution uncertainty is about $1 \%$, while the jet mass correction uncertainty is $10 \%$. The $H_{\mathrm{T}}$-reweighting has an uncertainty of $1-3 \%$ for the $A-D$ regions used in the background estimation.

The subjet $\mathrm{b}$ tagging and the $\mathrm{t}$ tagging scale factor uncertainties also affect the $M(\mathrm{~T})$ shape. The t tagging scale factor uncertainty is the largest at about $15-30 \%$ over the entire $p_{\mathrm{T}}$ range. The subjet $\mathrm{b}$ tagging scale factor systematic uncertainties are $2-5 \%$ for subjets from b quarks; they are a factor of two larger for c quarks, and about $10 \%$ for light quark and gluon subjets. As discussed in section 5, the systematic uncertainties in the estimated multijets background is anticorrelated with those for the simulated $t \bar{t}+$ jets, $W+$ jets, and tW backgrounds.

\section{Results}

We set limits on the product of the signal cross sections and the branching fraction $\mathcal{B}(\mathrm{T} \rightarrow \mathrm{tH})$ for the $\mathrm{T}$ quark produced in association with a top or a bottom through electroweak interactions. A binned likelihood fit to the data with the shapes of the $M(\mathrm{~T})$ candidate distributions for the background and the signal is made to obtain the $95 \%$ confidence level (CL) upper limit on the signal. The systematic uncertainties, treated as nuisance parameters in the likelihood function, are marginalized following a Bayesian approach [58, 59].

The expected and observed limits are shown in figure 4 for different $\mathrm{T}$ quark masses, and with LH and RH couplings of the T quark to the third-generation SM quarks. The limits are listed in table 4 . The cross section limits are derived with a signal sample simulated using the narrow width of $10 \mathrm{GeV}$. Studies on samples generated using larger widths have established that the reconstructed $M(\mathrm{~T})$ distributions do not change significantly compared to the narrow width approximation for T quarks having a width of up to $10 \%$ of their masses. The signal selection efficiency is estimated to decrease by about $7 \%$ for a T quark with a width of $10 \%$, which is well within the uncertainties of the measurement. Hence, the measured limits on the cross sections are valid within uncertainties for a $\mathrm{T}$ quark of width of up to $10 \%$.

A comparison is made with the Simplest Simplified Model for a singlet and a doublet $\mathrm{T}$ quark. The branching fractions for the singlet case are $\mathcal{B}(\mathrm{T} \rightarrow \mathrm{bW}): \mathcal{B}(\mathrm{T} \rightarrow \mathrm{tZ}): \mathcal{B}(\mathrm{T} \rightarrow$ $\mathrm{tH})=0.5: 0.25: 0.25$, while for the doublet, $\mathcal{B}(\mathrm{T} \rightarrow \mathrm{bW}): \mathcal{B}(\mathrm{T} \rightarrow \mathrm{tZ}): \mathcal{B}(\mathrm{T} \rightarrow \mathrm{tH})=0:$ $0.5: 0.5$. In the SSM, only the LH coupling $c_{\mathrm{L}}^{\mathrm{bW}}$ is allowed for the singlet scenario, while for the doublet case there is only the $\mathrm{RH}$ coupling $c_{\mathrm{R}}^{\mathrm{tZ}}$. The cross sections are calculated assuming $c_{\mathrm{L}}^{\mathrm{bW}}=0.5$ and $c_{\mathrm{R}}^{\mathrm{tZ}}=0.5$ for the singlet and doublet scenarios, respectively. The values of the coupling factors correspond to a relatively narrow width for the $\mathrm{T}$ quark over the mass range searched for. 

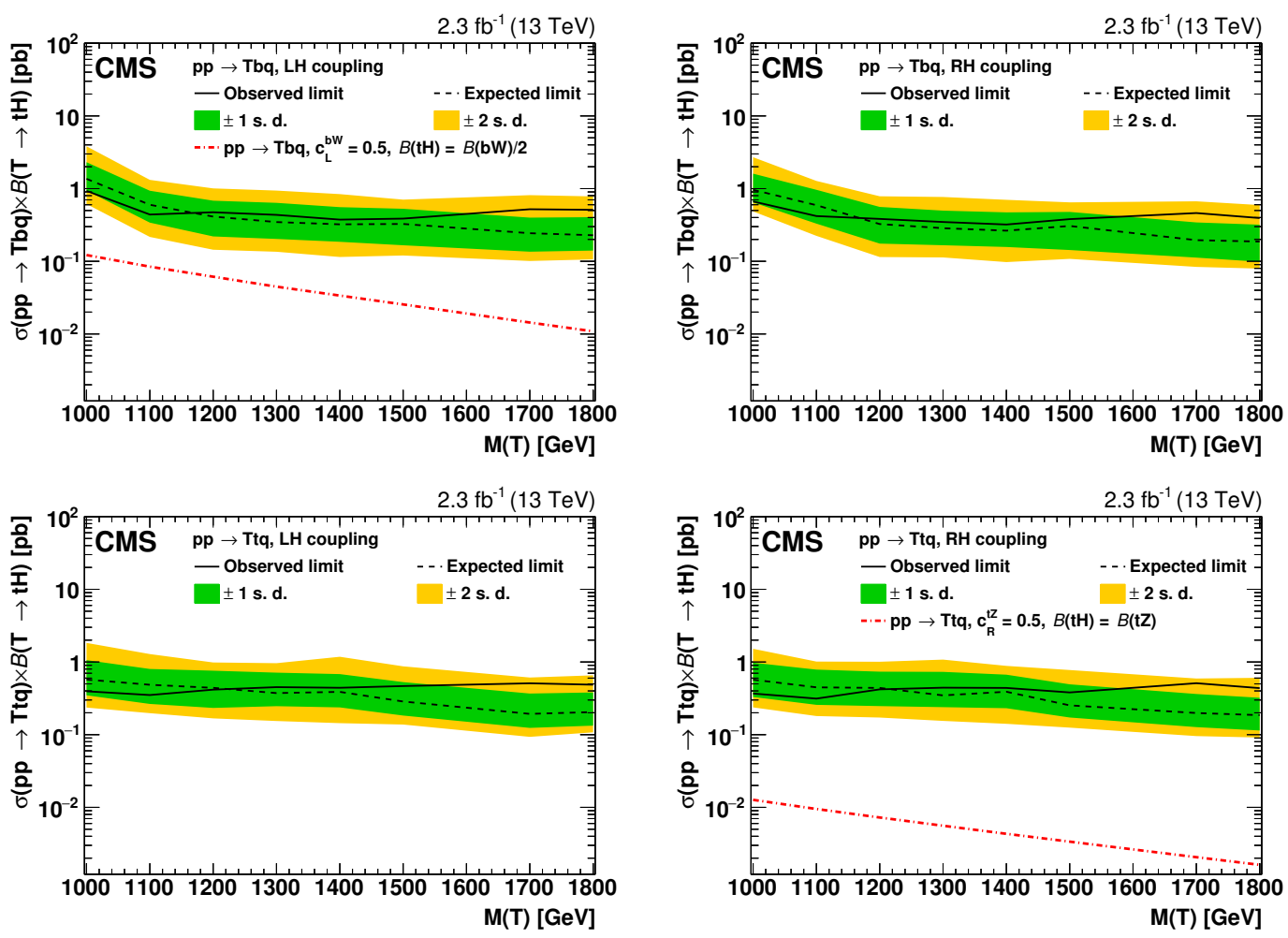

Figure 4. The expected and observed 95\% confidence level upper limits on the product of the signal cross section and the branching fraction $\mathcal{B}(\mathrm{T} \rightarrow \mathrm{tH})$ for the processes $\mathrm{pp} \rightarrow \mathrm{Tbq}$ (upper figures) and $\mathrm{pp} \rightarrow \mathrm{Ttq}$ (lower figures), for different assumed values of the T quark mass, and with left-handed (left figures) and right-handed (right figures) couplings to the standard model thirdgeneration quarks. The expected 1 and 2 standard deviation (s. d.) uncertainty bands are also shown. The limits are obtained assuming a resonance width of $10 \mathrm{GeV}$ for the $\mathrm{T}$ quark. The dotdashed curves in the upper left and lower right figures correspond to those predicted by the Simplest Simplified Model of refs. [18, 60], which predicts the existence of a left-handed and right-handed coupling for a singlet and doublet $\mathrm{T}$ quark, respectively. The benchmark coupling parameter values of $c_{\mathrm{L}}^{\mathrm{bW}}=0.5$ and $c_{\mathrm{R}}^{\mathrm{tZ}}=0.5$ are chosen for the comparison.

The SSM does not predict a RH singlet or a LH doublet, and thus theoretical curves are not shown for the upper right and the lower left plots of figure 4 . However, it should be noted that such couplings may still be possible in a non-minimal model. Furthermore, the observed limits on the cross sections correspond to values of the coupling factors that are larger than those associated with narrow resonances in the SSM. For a resonance width of $10 \%$ of the mass, which is the largest value for which the quoted limits are valid, the expected couplings lie between $0.6-0.3$ for a $\mathrm{T}$ quark of mass between $1000-1800 \mathrm{GeV}$.

\section{Summary}

A search for a vector-like top quark partner $\mathrm{T}$ in the single production mode is performed using proton-proton collision events at $\sqrt{s}=13 \mathrm{TeV}$ collected by the CMS experiment in 2015. The $\mathrm{T}$ quarks are assumed to couple only to the standard model third-generation 


\begin{tabular}{|c|c|c|c|c|c|c|c|c|}
\hline \multirow{3}{*}{ Mass $(\mathrm{GeV})$} & \multicolumn{2}{|c|}{$\mathrm{pp} \rightarrow \mathrm{Tbq}(\mathrm{LH})$} & \multicolumn{2}{|c|}{$\mathrm{pp} \rightarrow \mathrm{Tbq}(\mathrm{RH})$} & \multicolumn{2}{|c|}{$\mathrm{pp} \rightarrow \mathrm{Ttq}(\mathrm{LH})$} & \multicolumn{2}{|c|}{$\mathrm{pp} \rightarrow \mathrm{Ttq}(\mathrm{RH})$} \\
\hline & \multicolumn{8}{|c|}{ Limits in $\mathrm{pb}$} \\
\hline & Obs. & Exp. & Obs. & Exp. & Obs. & Exp. & Obs. & Exp. \\
\hline 1000 & 0.93 & 1.36 & 0.66 & 0.96 & 0.40 & 0.57 & 0.37 & 0.57 \\
\hline 1100 & 0.44 & 0.60 & 0.42 & 0.59 & 0.35 & 0.48 & 0.31 & 0.45 \\
\hline 1200 & 0.47 & 0.41 & 0.38 & 0.32 & 0.42 & 0.44 & 0.42 & 0.44 \\
\hline 1300 & 0.44 & 0.35 & 0.35 & 0.28 & 0.45 & 0.37 & 0.44 & 0.35 \\
\hline 1400 & 0.37 & 0.32 & 0.32 & 0.26 & 0.44 & 0.39 & 0.44 & 0.39 \\
\hline 1500 & 0.39 & 0.33 & 0.38 & 0.31 & 0.47 & 0.28 & 0.38 & 0.25 \\
\hline 1700 & 0.52 & 0.24 & 0.46 & 0.20 & 0.51 & 0.19 & 0.51 & 0.20 \\
\hline 1800 & 0.51 & 0.23 & 0.39 & 0.19 & 0.49 & 0.20 & 0.44 & 0.18 \\
\hline
\end{tabular}

Table 4. The observed and expected $95 \%$ confidence level upper limits on the product of the signal cross sections and the branching fraction $\mathcal{B}(\mathrm{T} \rightarrow \mathrm{tH})$, for various masses of the $\mathrm{T}$ quark.

quarks. The decay channel studied is $\mathrm{T} \rightarrow \mathrm{tH}$, with hadronic top quark decay and $\mathrm{H} \rightarrow \mathrm{b} \overline{\mathrm{b}}$. Boosted $\mathrm{H}$ and $\mathrm{t}$ tagging techniques are used to identify the Higgs boson and the top quark decays in the final state, and the invariant mass of the two gives the $\mathrm{T}$ quark candidate mass. The background is mostly due to the standard model $t \bar{t}+$ jets, with some contribution from multijet and $\mathrm{W}+$ jets processes. No significant excess of data above the background is observed in the $\mathrm{T}$ quark candidate mass distribution. The $95 \%$ confidence level upper limits on the product of the signal cross sections and the branching fraction $\mathcal{B}(\mathrm{T} \rightarrow \mathrm{tH})$ are set using Bayesian statistics. These vary between $0.31-0.93 \mathrm{pb}$ for a $\mathrm{T}$ quark of mass ranging from 1000 to $1800 \mathrm{GeV}$, in the $\mathrm{pp} \rightarrow \mathrm{Tbq}$ and $\mathrm{pp} \rightarrow$ Ttq production channels with left-handed and right-handed couplings to the standard model third-generation quarks. In the mass range considered for this analysis, the search sensitivity is essentially the same as that using leptonic final states [17]. The use of boosted techniques has led to an extension of the search region beyond those of previous analyses. This is the first time fully hadronic final states have been exploited in the search for single electroweak production of vector-like quarks at a hadron collider.

\section{Acknowledgments}

We congratulate our colleagues in the CERN accelerator departments for the excellent performance of the LHC and thank the technical and administrative staffs at CERN and at other CMS institutes for their contributions to the success of the CMS effort. In addition, we gratefully acknowledge the computing centers and personnel of the Worldwide LHC Computing Grid for delivering so effectively the computing infrastructure essential to our analyses. Finally, we acknowledge the enduring support for the construction and operation of the LHC and the CMS detector provided by the following funding agencies: BMWFW and FWF (Austria); FNRS and FWO (Belgium); CNPq, CAPES, FAPERJ, and FAPESP (Brazil); MES (Bulgaria); CERN; CAS, MoST, and NSFC (China); COLCIENCIAS (Colombia); MSES and CSF (Croatia); RPF (Cyprus); SENESCYT (Ecuador); MoER, ERC IUT, and ERDF (Estonia); Academy of Finland, MEC, and HIP (Finland); 
CEA and CNRS/IN2P3 (France); BMBF, DFG, and HGF (Germany); GSRT (Greece); OTKA and NIH (Hungary); DAE and DST (India); IPM (Iran); SFI (Ireland); INFN (Italy); MSIP and NRF (Republic of Korea); LAS (Lithuania); MOE and UM (Malaysia); BUAP, CINVESTAV, CONACYT, LNS, SEP, and UASLP-FAI (Mexico); MBIE (New Zealand); PAEC (Pakistan); MSHE and NSC (Poland); FCT (Portugal); JINR (Dubna); MON, RosAtom, RAS, RFBR and RAEP (Russia); MESTD (Serbia); SEIDI and CPAN (Spain); Swiss Funding Agencies (Switzerland); MST (Taipei); ThEPCenter, IPST, STAR, and NSTDA (Thailand); TUBITAK and TAEK (Turkey); NASU and SFFR (Ukraine); STFC (United Kingdom); DOE and NSF (U.S.A.).

Individuals have received support from the Marie-Curie program and the European Research Council and EPLANET (European Union); the Leventis Foundation; the A. P. Sloan Foundation; the Alexander von Humboldt Foundation; the Belgian Federal Science Policy Office; the Fonds pour la Formation à la Recherche dans l'Industrie et dans l'Agriculture (FRIA-Belgium); the Agentschap voor Innovatie door Wetenschap en Technologie (IWT-Belgium); the Ministry of Education, Youth and Sports (MEYS) of the Czech Republic; the Council of Science and Industrial Research, India; the HOMING PLUS program of the Foundation for Polish Science, cofinanced from European Union, Regional Development Fund, the Mobility Plus program of the Ministry of Science and Higher Education, the National Science Center (Poland), contracts Harmonia 2014/14/M/ST2/00428, Opus 2014/13/B/ST2/02543, 2014/15/B/ST2/03998, and 2015/19/B/ST2/02861, Sonatabis 2012/07/E/ST2/01406; the Thalis and Aristeia programs cofinanced by EU-ESF and the Greek NSRF; the National Priorities Research Program by Qatar National Research Fund; the Programa Clarín-COFUND del Principado de Asturias; the Rachadapisek Sompot Fund for Postdoctoral Fellowship, Chulalongkorn University and the Chulalongkorn Academic into Its 2nd Century Project Advancement Project (Thailand); and the Welch Foundation, contract C-1845.

Open Access. This article is distributed under the terms of the Creative Commons Attribution License (CC-BY 4.0), which permits any use, distribution and reproduction in any medium, provided the original author(s) and source are credited.

\section{References}

[1] M. Schmaltz and D. Tucker-Smith, Little Higgs review, Ann. Rev. Nucl. Part. Sci. 55 (2005) 229 [hep-ph/0502182] [INSPIRE].

[2] M. Perelstein, M.E. Peskin and A. Pierce, Top quarks and electroweak symmetry breaking in little Higgs models, Phys. Rev. D 69 (2004) 075002 [hep-ph/0310039] [INSPIRE].

[3] I. Antoniadis, K. Benakli and M. Quirós, Finite Higgs mass without supersymmetry, New J. Phys. 3 (2001) 20 [hep-th/0108005] [INSPIRE].

[4] Y. Hosotani, S. Noda and K. Takenaga, Dynamical gauge-Higgs unification in the electroweak theory, Phys. Lett. B 607 (2005) 276 [hep-ph/0410193] [inSPIRE].

[5] K. Agashe, R. Contino and A. Pomarol, The minimal composite Higgs model, Nucl. Phys. B 719 (2005) 165 [hep-ph/0412089] [INSPIRE]. 
[6] R. Contino, L. Da Rold and A. Pomarol, Light custodians in natural composite Higgs models, Phys. Rev. D 75 (2007) 055014 [hep-ph/0612048] [INSPIRE].

[7] P.H. Frampton, P.Q. Hung and M. Sher, Quarks and leptons beyond the third generation, Phys. Rept. 330 (2000) 263 [hep-ph/9903387] [INSPIRE].

[8] A. De Simone, O. Matsedonskyi, R. Rattazzi and A. Wulzer, A first top partner hunter's guide, JHEP 04 (2013) 004 [arXiv:1211.5663] [INSPIRE].

[9] Y. Okada and L. Panizzi, LHC signatures of vector-like quarks, Adv. High Energy Phys. 2013 (2013) 364936 [arXiv:1207.5607] [INSPIRE].

[10] ATLAS, CMS collaboration, Measurements of the Higgs boson production and decay rates and constraints on its couplings from a combined ATLAS and CMS analysis of the LHC pp collision data at $\sqrt{s}=7$ and $8 \mathrm{TeV}$, JHEP 08 (2016) 045 [arXiv:1606.02266] [INSPIRE].

[11] ATLAS collaboration, Search for production of vector-like quark pairs and of four top quarks in the lepton-plus-jets final state in pp collisions at $\sqrt{s}=8 \mathrm{TeV}$ with the ATLAS detector, JHEP 08 (2015) 105 [arXiv: 1505.04306] [INSPIRE].

[12] CMS collaboration, Search for vector-like charge $2 / 3$ T quarks in proton-proton collisions at $\sqrt{s}=8$ TeV, Phys. Rev. D 93 (2016) 012003 [arXiv: 1509.04177] [INSPIRE].

[13] ATLAS collaboration, Search for single production of vector-like quarks decaying into $\mathrm{Wb}$ in pp collisions at $\sqrt{s}=8 \mathrm{TeV}$ with the ATLAS detector, Eur. Phys. J. C 76 (2016) 442 [arXiv: 1602.05606] [INSPIRE].

[14] J.A. Aguilar-Saavedra, R. Benbrik, S. Heinemeyer and M. Pérez-Victoria, Handbook of vectorlike quarks: Mixing and single production, Phys. Rev. D 88 (2013) 094010 [arXiv: 1306.0572] [INSPIRE].

[15] ATLAS, CMS collaboration, G. Aad et al., Combined measurement of the Higgs boson mass in pp collisions at $\sqrt{s}=7$ and 8 TeV with the ATLAS and CMS experiments, Phys. Rev. Lett. 114 (2015) 191803 [arXiv:1503.07589] [INSPIRE].

[16] LHC Higgs Cross Section Working Group, D. de Florian et al., Handbook of LHC Higgs cross sections: 4. Deciphering the nature of the Higgs sector, arXiv:1610.07922.

[17] CMS collaboration, Search for single production of a heavy vector-like $T$ quark decaying to a Higgs boson and a top quark with a lepton and jets in the final state, submitted to, Phys. Lett. B (2016), arXiv:1612.00999 [INSPIRE].

[18] O. Matsedonskyi, G. Panico and A. Wulzer, On the interpretation of top partners searches, JHEP 12 (2014) 097 [arXiv: 1409.0100] [INSPIRE].

[19] J. Alwall et al., The automated computation of tree-level and next-to-leading order differential cross sections and their matching to parton shower simulations, JHEP 07 (2014) 079 [arXiv: 1405.0301] [INSPIRE].

[20] NNPDF collaboration, R.D. Ball et al., Parton distributions for the LHC Run II, JHEP 04 (2015) 040 [arXiv: 1410.8849] [INSPIRE].

[21] M. Czakon and A. Mitov, Top++: a program for the calculation of the top-pair cross-section at hadron colliders, Comput. Phys. Commun. 185 (2014) 2930 [arXiv:1112.5675] [INSPIRE].

[22] N. Kidonakis, Two-loop soft anomalous dimensions for single top quark associated production with a $W^{-}$or $H^{-}$, Phys. Rev. D 82 (2010) 054018 [arXiv: 1005.4451] [INSPIRE]. 
[23] N. Kidonakis, Top quark production, in the proceedings of the Helmholtz International Summer School on Physics of Heavy Quarks and Hadrons (HQ 2013), July 15-28, Dubna, Russia, DESY (2014), arXiv:1311.0283.

[24] P. Nason, A new method for combining NLO QCD with shower Monte Carlo algorithms, JHEP 11 (2004) 040 [hep-ph/0409146] [INSPIRE].

[25] S. Frixione, P. Nason and C. Oleari, Matching NLO QCD computations with Parton Shower simulations: the POWHEG method, JHEP 11 (2007) 070 [arXiv:0709.2092] [INSPIRE].

[26] S. Alioli, P. Nason, C. Oleari and E. Re, A general framework for implementing NLO calculations in shower Monte Carlo programs: the POWHEG BOX, JHEP 06 (2010) 043 [arXiv: 1002.2581] [INSPIRE].

[27] S. Frixione, P. Nason and G. Ridolfi, A positive-weight next-to-leading-order Monte Carlo for heavy flavour hadroproduction, JHEP 09 (2007) 126 [arXiv:0707.3088] [INSPIRE].

[28] Y. Li and F. Petriello, Combining QCD and electroweak corrections to dilepton production in FEWZ, Phys. Rev. D 86 (2012) 094034 [arXiv:1208.5967] [INSPIRE].

[29] T. Sjöstrand, S. Mrenna and P.Z. Skands, A brief introduction to PYTHIA 8.1, Comput. Phys. Commun. 178 (2008) 852 [arXiv:0710.3820] [INSPIRE].

[30] CMS collaboration, Event generator tunes obtained from underlying event and multiparton scattering measurements, Eur. Phys. J. C 76 (2016) 155 [arXiv:1512.00815] [INSPIRE].

[31] M.L. Mangano, M. Moretti, F. Piccinini and M. Treccani, Matching matrix elements and shower evolution for top-quark production in hadronic collisions, JHEP 01 (2007) 013 [hep-ph/0611129] [INSPIRE].

[32] ATLAS collaboration, Measurement of the inelastic proton-proton cross section at $\sqrt{s}=13$ TeV with the ATLAS detector at the LHC, Phys. Rev. Lett. 117 (2016) 182002 [arXiv: 1606.02625] [INSPIRE].

[33] J. Allison et al., GEANT4 developments and applications, IEEE Trans. Nucl. Sci. 53 (2006) 270.

[34] J. Allison et al., Recent developments in GEANT4, Nucl. Instrum. Meth. A 835 (2016) 186 [INSPIRE].

[35] CMS collaboration, The CMS experiment at the CERN LHC, 2008 JINST 3 S08004 [INSPIRE].

[36] CMS collaboration, Particle-flow event reconstruction in CMS and performance for jets, taus and MET, CMS-PAS-PFT-09-001 (2009).

[37] CMS Collaboration, Commissioning of the particle-flow event reconstruction with the first LHC collisions recorded in the CMS detector, CMS-PAS-PFT-10-001 (2010).

[38] M. Cacciari, G.P. Salam and G. Soyez, The anti- $k_{t}$ jet clustering algorithm, JHEP 04 (2008) 063 [arXiv: 0802.1189] [INSPIRE].

[39] M. Cacciari, G.P. Salam and G. Soyez, FastJet user manual, Eur. Phys. J. C 72 (2012) 1896 [arXiv:1111.6097] [INSPIRE].

[40] M. Cacciari and G.P. Salam, Dispelling the $N^{3}$ myth for the $k_{t}$ jet-finder, Phys. Lett. B 641 (2006) 57 [hep-ph/0512210] [INSPIRE].

[41] M. Cacciari and G.P. Salam, Pileup subtraction using jet areas, Phys. Lett. B 659 (2008) 119 [arXiv: 0707.1378] [INSPIRE]. 
[42] CMS collaboration, Determination of jet energy calibration and transverse momentum resolution in CMS, 2011 JINST 6 P11002 [arXiv:1107.4277] [INSPIRE].

[43] CMS collaboration, Jet energy scale and resolution in the CMS experiment in pp collisions at $8 \mathrm{TeV}, 2017$ JINST 12 P02014 [arXiv: 1607.03663] [INSPIRE].

[44] CMS collaboration, Jet performance in pp collisions at 7 TeV, CMS-PAS-JME-10-003 (2010).

[45] G.P. Salam, Towards Jetography, Eur. Phys. J. C 67 (2010) 637 [arXiv:0906.1833] [INSPIRE].

[46] S.D. Ellis, C.K. Vermilion and J.R. Walsh, Recombination algorithms and jet substructure: pruning as a tool for heavy particle searches, Phys. Rev. D 81 (2010) 094023 [arXiv:0912.0033] [INSPIRE].

[47] M. Dasgupta, A. Fregoso, S. Marzani and G.P. Salam, Towards an understanding of jet substructure, JHEP 09 (2013) 029 [arXiv: 1307.0007] [INSPIRE].

[48] A.J. Larkoski, S. Marzani, G. Soyez and J. Thaler, Soft drop, JHEP 05 (2014) 146 [arXiv: 1402 . 2657] [INSPIRE].

[49] CMS collaboration, $V$ tagging observables and correlations, CMS-PAS-JME-14-002 (2014).

[50] CMS collaboration, Top Tagging with New Approaches, CMS-PAS-JME-15-002 (2016).

[51] CMS collaboration, Identification techniques for highly boosted $W$ bosons that decay into hadrons, JHEP 12 (2014) 017 [arXiv:1410.4227] [INSPIRE].

[52] CMS collaboration, Boosted top jet tagging at CMS, CMS-PAS-JME-13-007 (2013).

[53] J. Thaler and K. Van Tilburg, Identifying boosted objects with N-subjettiness, JHEP 03 (2011) 015 [arXiv:1011.2268] [INSPIRE].

[54] CMS collaboration, Identification of b-quark jets with the CMS experiment, 2013 JINST 8 P04013 [arXiv: 1211.4462] [INSPIRE].

[55] CMS collaboration, Identification of b quark jets at the CMS Experiment in the LHC Run 2, CMS-PAS-BTV-15-001 (2015).

[56] CMS collaboration, CMS luminosity measurement for the 2015 data-taking period, CMS-PAS-LUM-15-001 (2015).

[57] J. Butterworth et al., PDF 4 LHC recommendations for LHC Run II, J. Phys. G 43 (2016) 023001 [arXiv: 1510.03865 ] [INSPIRE].

[58] D. Lindley, Kendall's advanced theory of statistics. Volume 2B: bayesian inference, 2nd edn., J, Roy. Stat. Soc. A 168 (2005) 259.

[59] ATLAS and CMS collaboration, and The LHC Higgs Combination Group, Procedure for the LHC Higgs boson search combination in Summer 2011, CMS-NOTE-2011-005 (2011).

[60] J.M. Campbell, R.K. Ellis and F. Tramontano, Single top production and decay at next-to-leading order, Phys. Rev. D 70 (2004) 094012 [hep-ph/0408158] [INSPIRE]. 


\section{The CMS collaboration}

\section{Yerevan Physics Institute, Yerevan, Armenia}

A.M. Sirunyan, A. Tumasyan

\section{Institut für Hochenergiephysik, Wien, Austria}

W. Adam, E. Asilar, T. Bergauer, J. Brandstetter, E. Brondolin, M. Dragicevic, J. Erö, M. Flechl, M. Friedl, R. Frühwirth ${ }^{1}$, V.M. Ghete, C. Hartl, N. Hörmann, J. Hrubec, M. Jeitler ${ }^{1}$, A. König, I. Krätschmer, D. Liko, T. Matsushita, I. Mikulec, D. Rabady, N. Rad, B. Rahbaran, H. Rohringer, J. Schieck ${ }^{1}$, J. Strauss, W. Waltenberger, C.-E. Wulz ${ }^{1}$

Institute for Nuclear Problems, Minsk, Belarus

V. Chekhovsky, O. Dvornikov, Y. Dydyshka, I. Emeliantchik, A. Litomin, V. Makarenko, V. Mossolov, R. Stefanovitch, J. Suarez Gonzalez, V. Zykunov

\section{National Centre for Particle and High Energy Physics, Minsk, Belarus}

N. Shumeiko

\section{Universiteit Antwerpen, Antwerpen, Belgium}

S. Alderweireldt, E.A. De Wolf, X. Janssen, J. Lauwers, M. Van De Klundert, H. Van Haevermaet, P. Van Mechelen, N. Van Remortel, A. Van Spilbeeck

\section{Vrije Universiteit Brussel, Brussel, Belgium}

S. Abu Zeid, F. Blekman, J. D'Hondt, N. Daci, I. De Bruyn, K. Deroover, S. Lowette, S. Moortgat, L. Moreels, A. Olbrechts, Q. Python, K. Skovpen, S. Tavernier, W. Van Doninck, P. Van Mulders, I. Van Parijs

\section{Université Libre de Bruxelles, Bruxelles, Belgium}

H. Brun, B. Clerbaux, G. De Lentdecker, H. Delannoy, G. Fasanella, L. Favart, R. Goldouzian, A. Grebenyuk, G. Karapostoli, T. Lenzi, A. Léonard, J. Luetic, T. Maerschalk, A. Marinov, A. Randle-conde, T. Seva, C. Vander Velde, P. Vanlaer, D. Vannerom, R. Yonamine, F. Zenoni, F. Zhang ${ }^{2}$

\section{Ghent University, Ghent, Belgium}

A. Cimmino, T. Cornelis, D. Dobur, A. Fagot, M. Gul, I. Khvastunov, D. Poyraz, S. Salva, R. Schöfbeck, M. Tytgat, W. Van Driessche, E. Yazgan, N. Zaganidis

Université Catholique de Louvain, Louvain-la-Neuve, Belgium

H. Bakhshiansohi, C. Beluffi ${ }^{3}$, O. Bondu, S. Brochet, G. Bruno, A. Caudron, S. De Visscher, C. Delaere, M. Delcourt, B. Francois, A. Giammanco, A. Jafari, M. Komm, G. Krintiras, V. Lemaitre, A. Magitteri, A. Mertens, M. Musich, C. Nuttens, K. Piotrzkowski, L. Quertenmont, M. Selvaggi, M. Vidal Marono, S. Wertz

\section{Université de Mons, Mons, Belgium}

N. Beliy

\section{Centro Brasileiro de Pesquisas Fisicas, Rio de Janeiro, Brazil}

W.L. Aldá Júnior, F.L. Alves, G.A. Alves, L. Brito, C. Hensel, A. Moraes, M.E. Pol, P. Rebello Teles 
Universidade do Estado do Rio de Janeiro, Rio de Janeiro, Brazil

E. Belchior Batista Das Chagas, W. Carvalho, J. Chinellato ${ }^{4}$, A. Custódio, E.M. Da Costa, G.G. Da Silveira ${ }^{5}$, D. De Jesus Damiao, C. De Oliveira Martins, S. Fonseca De Souza, L.M. Huertas Guativa, H. Malbouisson, D. Matos Figueiredo, C. Mora Herrera, L. Mundim, H. Nogima, W.L. Prado Da Silva, A. Santoro, A. Sznajder, E.J. Tonelli Manganote ${ }^{4}$, A. Vilela Pereira

Universidade Estadual Paulista ${ }^{a}$, Universidade Federal do ABC ${ }^{b}$, São Paulo, Brazil

S. Ahuja ${ }^{a}$, C.A. Bernardes ${ }^{a}$, S. Dogra ${ }^{a}$, T.R. Fernandez Perez Tomei $^{a}$, E.M. Gregores ${ }^{b}$, P.G. Mercadante ${ }^{b}$, C.S. Moon ${ }^{a}$, S.F. Novaes ${ }^{a}$, Sandra S. Padula ${ }^{a}$, D. Romero Abad ${ }^{b}$, J.C. Ruiz Vargas ${ }^{a}$

Institute for Nuclear Research and Nuclear Energy, Sofia, Bulgaria

A. Aleksandrov, R. Hadjiiska, P. Iaydjiev, M. Rodozov, S. Stoykova, G. Sultanov, M. Vutova

University of Sofia, Sofia, Bulgaria

A. Dimitrov, I. Glushkov, L. Litov, B. Pavlov, P. Petkov

Beihang University, Beijing, China

W. Fang ${ }^{6}$

Institute of High Energy Physics, Beijing, China

M. Ahmad, J.G. Bian, G.M. Chen, H.S. Chen, M. Chen, Y. Chen ${ }^{7}$, T. Cheng, C.H. Jiang,

D. Leggat, Z. Liu, F. Romeo, M. Ruan, S.M. Shaheen, A. Spiezia, J. Tao, C. Wang,

Z. Wang, H. Zhang, J. Zhao

State Key Laboratory of Nuclear Physics and Technology, Peking University, Beijing, China

Y. Ban, G. Chen, Q. Li, S. Liu, Y. Mao, S.J. Qian, D. Wang, Z. Xu

Universidad de Los Andes, Bogota, Colombia

C. Avila, A. Cabrera, L.F. Chaparro Sierra, C. Florez, J.P. Gomez, C.F. González Hernández, J.D. Ruiz Alvarez, J.C. Sanabria

University of Split, Faculty of Electrical Engineering, Mechanical Engineering and Naval Architecture, Split, Croatia

N. Godinovic, D. Lelas, I. Puljak, P.M. Ribeiro Cipriano, T. Sculac

University of Split, Faculty of Science, Split, Croatia

Z. Antunovic, M. Kovac

Institute Rudjer Boskovic, Zagreb, Croatia

V. Brigljevic, D. Ferencek, K. Kadija, B. Mesic, S. Micanovic, L. Sudic, T. Susa

University of Cyprus, Nicosia, Cyprus

A. Attikis, G. Mavromanolakis, J. Mousa, C. Nicolaou, F. Ptochos, P.A. Razis, H. Rykaczewski, D. Tsiakkouri 
Charles University, Prague, Czech Republic

M. Finger ${ }^{8}$, M. Finger Jr. ${ }^{8}$

Universidad San Francisco de Quito, Quito, Ecuador

E. Carrera Jarrin

Academy of Scientific Research and Technology of the Arab Republic of Egypt, Egyptian Network of High Energy Physics, Cairo, Egypt

A. Ellithi Kamel ${ }^{9}$, M.A. Mahmoud ${ }^{10,11}$, A. Radi ${ }^{11,12}$

National Institute of Chemical Physics and Biophysics, Tallinn, Estonia

M. Kadastik, L. Perrini, M. Raidal, A. Tiko, C. Veelken

Department of Physics, University of Helsinki, Helsinki, Finland

P. Eerola, J. Pekkanen, M. Voutilainen

Helsinki Institute of Physics, Helsinki, Finland

J. Härkönen, T. Järvinen, V. Karimäki, R. Kinnunen, T. Lampén, K. Lassila-Perini,

S. Lehti, T. Lindén, P. Luukka, J. Tuominiemi, E. Tuovinen, L. Wendland

Lappeenranta University of Technology, Lappeenranta, Finland

J. Talvitie, T. Tuuva

IRFU, CEA, Université Paris-Saclay, Gif-sur-Yvette, France

M. Besancon, F. Couderc, M. Dejardin, D. Denegri, B. Fabbro, J.L. Faure, C. Favaro, F. Ferri, S. Ganjour, S. Ghosh, A. Givernaud, P. Gras, G. Hamel de Monchenault, P. Jarry, I. Kucher, E. Locci, M. Machet, J. Malcles, J. Rander, A. Rosowsky, M. Titov, A. Zghiche

Laboratoire Leprince-Ringuet, Ecole Polytechnique, IN2P3-CNRS, Palaiseau, France

A. Abdulsalam, I. Antropov, S. Baffioni, F. Beaudette, P. Busson, L. Cadamuro, E. Chapon, C. Charlot, O. Davignon, R. Granier de Cassagnac, M. Jo, S. Lisniak, P. Miné, M. Nguyen, C. Ochando, G. Ortona, P. Paganini, P. Pigard, S. Regnard, R. Salerno, Y. Sirois, T. Strebler, Y. Yilmaz, A. Zabi

Institut Pluridisciplinaire Hubert Curien (IPHC), Université de Strasbourg, CNRS-IN2P3

J.-L. Agram ${ }^{13}$, J. Andrea, A. Aubin, D. Bloch, J.-M. Brom, M. Buttignol, E.C. Chabert, N. Chanon, C. Collard, E. Conte ${ }^{13}$, X. Coubez, J.-C. Fontaine ${ }^{13}$, D. Gelé, U. Goerlach, A.-C. Le Bihan, P. Van Hove

Centre de Calcul de l'Institut National de Physique Nucleaire et de Physique des Particules, CNRS/IN2P3, Villeurbanne, France

S. Gadrat

Université de Lyon, Université Claude Bernard Lyon 1, CNRS-IN2P3, Institut de Physique Nucléaire de Lyon, Villeurbanne, France

S. Beauceron, C. Bernet, G. Boudoul, C.A. Carrillo Montoya, R. Chierici, D. Contardo, B. Courbon, P. Depasse, H. El Mamouni, J. Fan, J. Fay, S. Gascon, M. Gouzevitch, 
G. Grenier, B. Ille, F. Lagarde, I.B. Laktineh, M. Lethuillier, L. Mirabito, A.L. Pequegnot, S. Perries, A. Popov ${ }^{14}$, D. Sabes, V. Sordini, M. Vander Donckt, P. Verdier, S. Viret

\section{Georgian Technical University, Tbilisi, Georgia}

A. Khvedelidze ${ }^{8}$

Tbilisi State University, Tbilisi, Georgia

Z. Tsamalaidze ${ }^{8}$

RWTH Aachen University, I. Physikalisches Institut, Aachen, Germany

C. Autermann, S. Beranek, L. Feld, M.K. Kiesel, K. Klein, M. Lipinski, M. Preuten, C. Schomakers, J. Schulz, T. Verlage

RWTH Aachen University, III. Physikalisches Institut A, Aachen, Germany A. Albert, M. Brodski, E. Dietz-Laursonn, D. Duchardt, M. Endres, M. Erdmann, S. Erdweg, T. Esch, R. Fischer, A. Güth, M. Hamer, T. Hebbeker, C. Heidemann, K. Hoepfner, S. Knutzen, M. Merschmeyer, A. Meyer, P. Millet, S. Mukherjee, M. Olschewski, K. Padeken, T. Pook, M. Radziej, H. Reithler, M. Rieger, F. Scheuch, L. Sonnenschein, D. Teyssier, S. Thüer

RWTH Aachen University, III. Physikalisches Institut B, Aachen, Germany

V. Cherepanov, G. Flügge, B. Kargoll, T. Kress, A. Künsken, J. Lingemann, T. Müller, A. Nehrkorn, A. Nowack, C. Pistone, O. Pooth, A. Stahl ${ }^{15}$

\section{Deutsches Elektronen-Synchrotron, Hamburg, Germany}

M. Aldaya Martin, T. Arndt, C. Asawatangtrakuldee, K. Beernaert, O. Behnke, U. Behrens, A.A. Bin Anuar, K. Borras ${ }^{16}$, A. Campbell, P. Connor, C. ContrerasCampana, F. Costanza, C. Diez Pardos, G. Dolinska, G. Eckerlin, D. Eckstein, T. Eichhorn, E. Eren, E. Gallo ${ }^{17}$, J. Garay Garcia, A. Geiser, A. Gizhko, J.M. Grados Luyando, A. Grohsjean, P. Gunnellini, A. Harb, J. Hauk, M. Hempel ${ }^{18}$, H. Jung, A. Kalogeropoulos, O. Karacheban ${ }^{18}$, M. Kasemann, J. Keaveney, C. Kleinwort, I. Korol, D. Krücker, W. Lange, A. Lelek, J. Leonard, K. Lipka, A. Lobanov, W. Lohmann ${ }^{18}$, R. Mankel, I.A. Melzer-Pellmann, A.B. Meyer, G. Mittag, J. Mnich, A. Mussgiller, E. Ntomari, D. Pitzl, R. Placakyte, A. Raspereza, B. Roland, M.Ö. Sahin, P. Saxena, T. Schoerner-Sadenius, C. Seitz, S. Spannagel, N. Stefaniuk, G.P. Van Onsem, R. Walsh, C. Wissing

\section{University of Hamburg, Hamburg, Germany}

V. Blobel, M. Centis Vignali, A.R. Draeger, T. Dreyer, E. Garutti, D. Gonzalez, J. Haller, M. Hoffmann, A. Junkes, R. Klanner, R. Kogler, N. Kovalchuk, T. Lapsien, T. Lenz, I. Marchesini, D. Marconi, M. Meyer, M. Niedziela, D. Nowatschin, F. Pantaleo ${ }^{15}$, T. Peiffer, A. Perieanu, J. Poehlsen, C. Sander, C. Scharf, P. Schleper, A. Schmidt, S. Schumann, J. Schwandt, H. Stadie, G. Steinbrück, F.M. Stober, M. Stöver, H. Tholen, D. Troendle, E. Usai, L. Vanelderen, A. Vanhoefer, B. Vormwald

Institut für Experimentelle Kernphysik, Karlsruhe, Germany

M. Akbiyik, C. Barth, S. Baur, C. Baus, J. Berger, E. Butz, R. Caspart, T. Chwalek, F. Colombo, W. De Boer, A. Dierlamm, S. Fink, B. Freund, R. Friese, M. Giffels, A. Gilbert, 
P. Goldenzweig, D. Haitz, F. Hartmann ${ }^{15}$, S.M. Heindl, U. Husemann, I. Katkov ${ }^{14}$, S. Kudella, H. Mildner, M.U. Mozer, Th. Müller, M. Plagge, G. Quast, K. Rabbertz, S. Röcker, F. Roscher, M. Schröder, I. Shvetsov, G. Sieber, H.J. Simonis, R. Ulrich, S. Wayand, M. Weber, T. Weiler, S. Williamson, C. Wöhrmann, R. Wolf

Institute of Nuclear and Particle Physics (INPP), NCSR Demokritos, Aghia Paraskevi, Greece

G. Anagnostou, G. Daskalakis, T. Geralis, V.A. Giakoumopoulou, A. Kyriakis, D. Loukas, I. Topsis-Giotis

National and Kapodistrian University of Athens, Athens, Greece

S. Kesisoglou, A. Panagiotou, N. Saoulidou, E. Tziaferi

University of Ioánnina, Ioánnina, Greece

I. Evangelou, G. Flouris, C. Foudas, P. Kokkas, N. Loukas, N. Manthos, I. Papadopoulos, E. Paradas

MTA-ELTE Lendület CMS Particle and Nuclear Physics Group, Eötvös Loránd University, Budapest, Hungary

N. Filipovic

Wigner Research Centre for Physics, Budapest, Hungary

G. Bencze, C. Hajdu, D. Horvath ${ }^{19}$, F. Sikler, V. Veszpremi, G. Vesztergombi ${ }^{20}$, A.J. Zsigmond

Institute of Nuclear Research ATOMKI, Debrecen, Hungary

N. Beni, S. Czellar, J. Karancsi21 ${ }^{21}$ A. Makovec, J. Molnar, Z. Szillasi

Institute of Physics, University of Debrecen

M. Bartók ${ }^{20}$, P. Raics, Z.L. Trocsanyi, B. Ujvari

National Institute of Science Education and Research, Bhubaneswar, India

S. Bahinipati, S. Choudhury ${ }^{22}$, P. Mal, K. Mandal, A. Nayak ${ }^{23}$, D.K. Sahoo, N. Sahoo, S.K. Swain

Panjab University, Chandigarh, India

S. Bansal, S.B. Beri, V. Bhatnagar, R. Chawla, U.Bhawandeep, A.K. Kalsi, A. Kaur, M. Kaur, R. Kumar, P. Kumari, A. Mehta, M. Mittal, J.B. Singh, G. Walia

University of Delhi, Delhi, India

Ashok Kumar, A. Bhardwaj, B.C. Choudhary, R.B. Garg, S. Keshri, S. Malhotra, M. Naimuddin, N. Nishu, K. Ranjan, R. Sharma, V. Sharma

Saha Institute of Nuclear Physics, Kolkata, India

R. Bhattacharya, S. Bhattacharya, K. Chatterjee, S. Dey, S. Dutt, S. Dutta, S. Ghosh, N. Majumdar, A. Modak, K. Mondal, S. Mukhopadhyay, S. Nandan, A. Purohit, A. Roy, D. Roy, S. Roy Chowdhury, S. Sarkar, M. Sharan, S. Thakur

Indian Institute of Technology Madras, Madras, India P.K. Behera 
Bhabha Atomic Research Centre, Mumbai, India

R. Chudasama, D. Dutta, V. Jha, V. Kumar, A.K. Mohanty ${ }^{15}$, P.K. Netrakanti, L.M. Pant, P. Shukla, A. Topkar

Tata Institute of Fundamental Research-A, Mumbai, India

T. Aziz, S. Dugad, G. Kole, B. Mahakud, S. Mitra, G.B. Mohanty, B. Parida, N. Sur, B. Sutar

Tata Institute of Fundamental Research-B, Mumbai, India

S. Banerjee, S. Bhowmik ${ }^{24}$, R.K. Dewanjee, S. Ganguly, M. Guchait, Sa. Jain, S. Kumar, M. Maity ${ }^{24}$, G. Majumder, K. Mazumdar, T. Sarkar ${ }^{24}$, N. Wickramage ${ }^{25}$

Indian Institute of Science Education and Research (IISER), Pune, India

S. Chauhan, S. Dube, V. Hegde, A. Kapoor, K. Kothekar, S. Pandey, A. Rane, S. Sharma

Institute for Research in Fundamental Sciences (IPM), Tehran, Iran

S. Chenarani ${ }^{26}$, E. Eskandari Tadavani, S.M. Etesami ${ }^{26}$, M. Khakzad, M. Mohammadi Najafabadi, M. Naseri, S. Paktinat Mehdiabadi ${ }^{27}$, F. Rezaei Hosseinabadi, B. Safarzadeh ${ }^{28}$, M. Zeinali

University College Dublin, Dublin, Ireland

M. Felcini, M. Grunewald

INFN Sezione di Bari ${ }^{a}$, Università di Bari ${ }^{b}$, Politecnico di Bari ${ }^{c}$, Bari, Italy

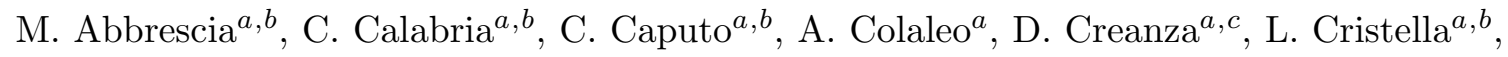
N. De Filippis ${ }^{a, c}$, M. De Palma ${ }^{a, b}$, L. Fiore ${ }^{a}$, G. Iaselli ${ }^{a, c}$, G. Maggi ${ }^{a, c}$, M. Maggi $^{a}$, G. Miniello ${ }^{a, b}$, S. My ${ }^{a, b}$, S. Nuzzo ${ }^{a, b}$, A. Pompili ${ }^{a, b}$, G. Pugliese ${ }^{a, c}$, R. Radogna ${ }^{a, b}$, A. Ranieri ${ }^{a}$, G. Selvaggi ${ }^{a, b}$, A. Sharma ${ }^{a}$, L. Silvestris ${ }^{a, 15}$, R. Venditti ${ }^{a, b}$, P. Verwilligen $^{a}$

INFN Sezione di Bologna ${ }^{a}$, Università di Bologna ${ }^{b}$, Bologna, Italy

G. Abbiendi ${ }^{a}$, C. Battilana, D. Bonacorsi ${ }^{a, b}$, S. Braibant-Giacomelli ${ }^{a, b}$, L. Brigliadori $^{a, b}$, R. Campanini ${ }^{a, b}$, P. Capiluppi ${ }^{a, b}$, A. Castro ${ }^{a, b}$, F.R. Cavallo $^{a}$, S.S. Chhibra ${ }^{a, b}$, G. Codispoti ${ }^{a, b}$, M. Cuffiani ${ }^{a, b}$, G.M. Dallavalle ${ }^{a}$, F. Fabbri $^{a}$, A. Fanfani ${ }^{a, b}$, D. Fasanella ${ }^{a, b}$, P. Giacomelli ${ }^{a}$, C. Grandi ${ }^{a}$, L. Guiducci ${ }^{a}, b$, S. Marcellini ${ }^{a}$, G. Masetti ${ }^{a}$, A. Montanari ${ }^{a}$,

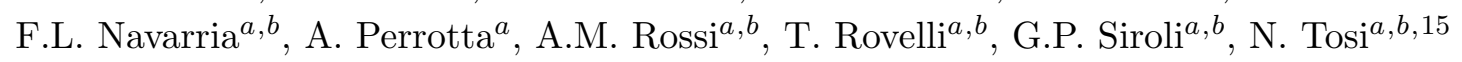

INFN Sezione di Catania ${ }^{a}$, Università di Catania ${ }^{b}$, Catania, Italy

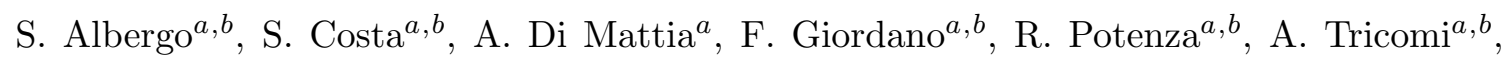
C. Tuve ${ }^{a, b}$

INFN Sezione di Firenze ${ }^{a}$, Università di Firenze ${ }^{b}$, Firenze, Italy

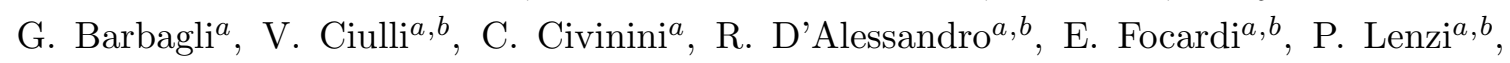
M. Meschini ${ }^{a}$, S. Paoletti ${ }^{a}$, G. Sguazzoni ${ }^{a}$, L. Viliani ${ }^{a, b, 15}$

INFN Laboratori Nazionali di Frascati, Frascati, Italy

L. Benussi, S. Bianco, F. Fabbri, D. Piccolo, F. Primavera ${ }^{15}$

INFN Sezione di Genova ${ }^{a}$, Università di Genova ${ }^{b}$, Genova, Italy

V. Calvelli ${ }^{a, b}$, F. Ferro ${ }^{a}$, M.R. Monge ${ }^{a, b}$, E. Robutti ${ }^{a}$, S. Tosi ${ }^{a, b}$ 
INFN Sezione di Milano-Bicocca ${ }^{a}$, Università di Milano-Bicocca ${ }^{b}$, Milano, Italy

L. Brianza ${ }^{a, b, 15}$, F. Brivio ${ }^{a, b}$, V. Ciriolo, M.E. Dinardo ${ }^{a, b}$, S. Fiorendi ${ }^{a, b, 15}$, S. Gennai ${ }^{a}$,

A. Ghezzi ${ }^{a, b}$, P. Govoni ${ }^{a, b}$, M. Malberti ${ }^{a, b}$, S. Malvezzi ${ }^{a}$, R.A. Manzoni ${ }^{a, b}$, D. Menasce ${ }^{a}$,

L. Moroni ${ }^{a}$, M. Paganoni ${ }^{a}, b$, D. Pedrini ${ }^{a}$, S. Pigazzini ${ }^{a, b}$, S. Ragazzi ${ }^{a, b}$, T. Tabarelli de Fatis $^{a, b}$

INFN Sezione di Napoli ${ }^{a}$, Università di Napoli 'Federico II' ${ }^{b}$, Napoli, Italy, Università della Basilicata ${ }^{c}$, Potenza, Italy, Università G. Marconi ${ }^{d}$, Roma, Italy

S. Buontempo ${ }^{a}$, N. Cavallo $^{a, c}$, G. De Nardo, S. Di Guida ${ }^{a, d, 15}$, M. Esposito ${ }^{a, b}$,

F. Fabozzi ${ }^{a, c}$, F. Fienga ${ }^{a, b}$, A.O.M. Iorio ${ }^{a, b}$, G. Lanza ${ }^{a}$, L. Lista ${ }^{a}$, S. Meola ${ }^{a, d, 15}$, P. Paolucci ${ }^{a, 15}$, C. Sciacca ${ }^{a, b}$, F. Thyssen ${ }^{a}$

INFN Sezione di Padova ${ }^{a}$, Università di Padova ${ }^{b}$, Padova, Italy, Università di Trento ${ }^{c}$, Trento, Italy

P. Azzi ${ }^{a, 15}$, N. Bacchetta ${ }^{a}$, L. Benato ${ }^{a, b}$, D. Bisello ${ }^{a, b}$, A. Boletti ${ }^{a, b}$, R. Carlin ${ }^{a, b}$, A. Carvalho Antunes De Oliveira ${ }^{a, b}$, P. Checchia ${ }^{a}$, M. Dall'Osso ${ }^{a, b}$, P. De Castro Manzano ${ }^{a}$, T. Dorigo ${ }^{a}$, U. Dosselli ${ }^{a}$, F. Gasparini ${ }^{a, b}$, U. Gasparini ${ }^{a}, b$, A. Gozzelino ${ }^{a}$, S. Lacaprara ${ }^{a}$, M. Margoni ${ }^{a, b}$, A.T. Meneguzzo ${ }^{a, b}$, J. Pazzini ${ }^{a, b}$, N. Pozzobon ${ }^{a, b}$, P. Ronchese ${ }^{a, b}$,

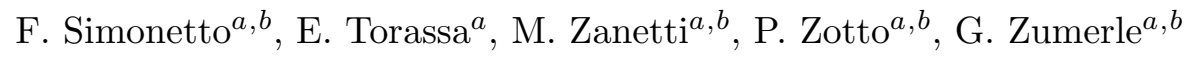

INFN Sezione di Pavia ${ }^{a}$, Università di Pavia ${ }^{b}$, Pavia, Italy

A. Braghieri ${ }^{a}$, A. Magnani ${ }^{a, b}$, P. Montagna ${ }^{a, b}$, S.P. Ratti ${ }^{a, b}$, V. Re ${ }^{a}$, C. Riccardi ${ }^{a, b}$, P. Salvini ${ }^{a}$, I. Vai ${ }^{a, b}$, P. Vitulo ${ }^{a, b}$

INFN Sezione di Perugia ${ }^{a}$, Università di Perugia ${ }^{b}$, Perugia, Italy

L. Alunni Solestizi ${ }^{a}, b$, G.M. Bilei ${ }^{a}$, D. Ciangottini ${ }^{a, b}$, L. Fanò ${ }^{a, b}$, P. Lariccia $^{a, b}$, R. Leonardi ${ }^{a, b}$, G. Mantovani ${ }^{a, b}$, M. Menichelli ${ }^{a}$, A. Saha ${ }^{a}$, A. Santocchia ${ }^{a, b}$

INFN Sezione di Pisa ${ }^{a}$, Università di Pisa ${ }^{b}$, Scuola Normale Superiore di Pisa ${ }^{c}$, Pisa, Italy

K. Androsov ${ }^{a, 29}$, P. Azzurri ${ }^{a, 15}$, G. Bagliesi ${ }^{a}$, J. Bernardini ${ }^{a}$, T. Boccali ${ }^{a}$, R. Castaldi $^{a}$, M.A. $\operatorname{Ciocci}^{a}{ }^{, 29}$, R. Dell'Orso ${ }^{a}$, S. Donato ${ }^{a, c}$, G. Fedi, A. Giassi ${ }^{a}$, M.T. Grippo ${ }^{a, 29}$,

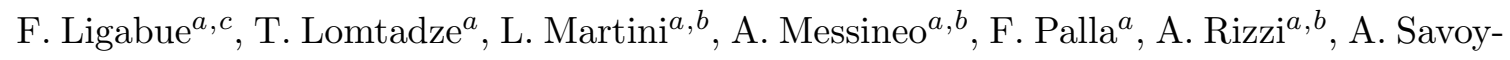

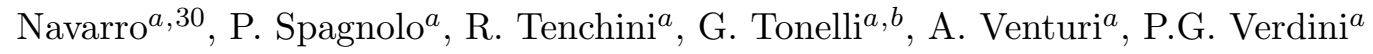

INFN Sezione di Roma ${ }^{a}$, Università di Roma ${ }^{b}$, Roma, Italy

L. Barone ${ }^{a, b}$, F. Cavallari ${ }^{a}$, M. Cipriani ${ }^{a, b}$, D. Del Re ${ }^{a, b, 15}$, M. Diemoz ${ }^{a}$, S. Gelli ${ }^{a, b}$, E. Longo $^{a, b}$, F. Margaroli ${ }^{a, b}$, B. Marzocchi ${ }^{a, b}$, P. Meridiani ${ }^{a}$, G. Organtini ${ }^{a, b}$,

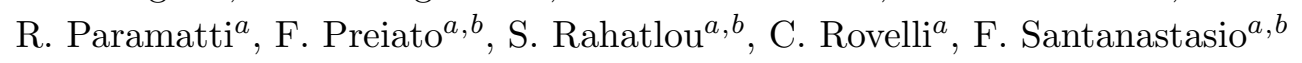

INFN Sezione di Torino ${ }^{a}$, Università di Torino ${ }^{b}$, Torino, Italy, Università del Piemonte Orientale ${ }^{c}$, Novara, Italy

N. Amapane ${ }^{a, b}$, R. Arcidiacono ${ }^{a, c, 15}$, S. Argiro ${ }^{a, b}$, M. Arneodo ${ }^{a, c}$, N. Bartosik ${ }^{a}$, R. Bellan ${ }^{a, b}$, C. Biino ${ }^{a}$, N. Cartiglia ${ }^{a}$, F. Cenna ${ }^{a, b}$, M. Costa ${ }^{a, b}$, R. Covarelli ${ }^{a, b}$, A. Degano ${ }^{a, b}$, N. Demaria ${ }^{a}$, L. Finco ${ }^{a, b}$, B. Kiani ${ }^{a, b}$, C. Mariotti ${ }^{a}$, S. Maselli ${ }^{a}$, 


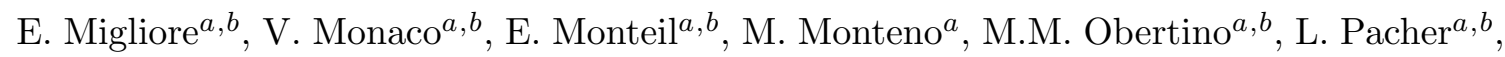
N. Pastrone $^{a}$, M. Pelliccioni ${ }^{a}$, G.L. Pinna Angioni ${ }^{a, b}$, F. Ravera ${ }^{a, b}$, A. Romero ${ }^{a, b}$, M. Ruspa ${ }^{a, c}$, R. Sacchi ${ }^{a, b}$, K. Shchelina ${ }^{a, b}$, V. Sola ${ }^{a}$, A. Solano ${ }^{a, b}$, A. Staiano ${ }^{a}$, P. Traczyk ${ }^{a, b}$

INFN Sezione di Trieste ${ }^{a}$, Università di Trieste ${ }^{b}$, Trieste, Italy

S. Belforte ${ }^{a}$, M. Casarsa ${ }^{a}$, F. Cossutti ${ }^{a}$, G. Della Ricca ${ }^{a}, b$, A. Zanetti ${ }^{a}$

Kyungpook National University, Daegu, Korea

D.H. Kim, G.N. Kim, M.S. Kim, S. Lee, S.W. Lee, Y.D. Oh, S. Sekmen, D.C. Son, Y.C. Yang

Chonbuk National University, Jeonju, Korea

A. Lee

Chonnam National University, Institute for Universe and Elementary Particles, Kwangju, Korea

H. Kim

Hanyang University, Seoul, Korea

J.A. Brochero Cifuentes, T.J. Kim

Korea University, Seoul, Korea

S. Cho, S. Choi, Y. Go, D. Gyun, S. Ha, B. Hong, Y. Jo, Y. Kim, K. Lee, K.S. Lee, S. Lee, J. Lim, S.K. Park, Y. Roh

Seoul National University, Seoul, Korea

J. Almond, J. Kim, H. Lee, S.B. Oh, B.C. Radburn-Smith, S.h. Seo, U.K. Yang, H.D. Yoo, G.B. Yu

University of Seoul, Seoul, Korea

M. Choi, H. Kim, J.H. Kim, J.S.H. Lee, I.C. Park, G. Ryu, M.S. Ryu

Sungkyunkwan University, Suwon, Korea

Y. Choi, J. Goh, C. Hwang, J. Lee, I. Yu

Vilnius University, Vilnius, Lithuania

V. Dudenas, A. Juodagalvis, J. Vaitkus

National Centre for Particle Physics, Universiti Malaya, Kuala Lumpur, Malaysia

I. Ahmed, Z.A. Ibrahim, J.R. Komaragiri, M.A.B. Md Ali ${ }^{31}$, F. Mohamad Idris ${ }^{32}$, W.A.T. Wan Abdullah, M.N. Yusli, Z. Zolkapli

Centro de Investigacion y de Estudios Avanzados del IPN, Mexico City, Mexico

H. Castilla-Valdez, E. De La Cruz-Burelo, I. Heredia-De La Cruz ${ }^{33}$, A. Hernandez-Almada,

R. Lopez-Fernandez, R. Magaña Villalba, J. Mejia Guisao, A. Sanchez-Hernandez

Universidad Iberoamericana, Mexico City, Mexico

S. Carrillo Moreno, C. Oropeza Barrera, F. Vazquez Valencia 
Benemerita Universidad Autonoma de Puebla, Puebla, Mexico

S. Carpinteyro, I. Pedraza, H.A. Salazar Ibarguen, C. Uribe Estrada

Universidad Autónoma de San Luis Potosí, San Luis Potosí, Mexico

A. Morelos Pineda

University of Auckland, Auckland, New Zealand

D. Krofcheck

University of Canterbury, Christchurch, New Zealand

P.H. Butler

National Centre for Physics, Quaid-I-Azam University, Islamabad, Pakistan

A. Ahmad, M. Ahmad, Q. Hassan, H.R. Hoorani, W.A. Khan, A. Saddique, M.A. Shah,

M. Shoaib, M. Waqas

National Centre for Nuclear Research, Swierk, Poland

H. Bialkowska, M. Bluj, B. Boimska, T. Frueboes, M. Górski, M. Kazana, K. Nawrocki,

K. Romanowska-Rybinska, M. Szleper, P. Zalewski

Institute of Experimental Physics, Faculty of Physics, University of Warsaw, Warsaw, Poland

K. Bunkowski, A. Byszuk ${ }^{34}$, K. Doroba, A. Kalinowski, M. Konecki, J. Krolikowski, M. Misiura, M. Olszewski, M. Walczak

Laboratório de Instrumentação e Física Experimental de Partículas, Lisboa, Portugal

P. Bargassa, C. Beirão Da Cruz E Silva, B. Calpas, A. Di Francesco, P. Faccioli, P.G. Ferreira Parracho, M. Gallinaro, J. Hollar, N. Leonardo, L. Lloret Iglesias, M.V. Nemallapudi, J. Rodrigues Antunes, J. Seixas, O. Toldaiev, D. Vadruccio, J. Varela, P. Vischia

Joint Institute for Nuclear Research, Dubna, Russia

S. Afanasiev, P. Bunin, M. Gavrilenko, I. Golutvin, A. Kamenev, V. Karjavin, A. Lanev, A. Malakhov, V. Matveev ${ }^{35,36}$, V. Palichik, V. Perelygin, M. Savina, S. Shmatov, S. Shulha,

N. Skatchkov, V. Smirnov, N. Voytishin, A. Zarubin

Petersburg Nuclear Physics Institute, Gatchina (St. Petersburg), Russia

L. Chtchipounov, V. Golovtsov, Y. Ivanov, V. Kim ${ }^{37}$, E. Kuznetsova ${ }^{38}$, V. Murzin, V. Oreshkin, V. Sulimov, A. Vorobyev

Institute for Nuclear Research, Moscow, Russia

Yu. Andreev, A. Dermenev, S. Gninenko, N. Golubev, A. Karneyeu, M. Kirsanov, N. Krasnikov, A. Pashenkov, D. Tlisov, A. Toropin

Institute for Theoretical and Experimental Physics, Moscow, Russia

V. Epshteyn, V. Gavrilov, N. Lychkovskaya, V. Popov, I. Pozdnyakov, G. Safronov, A. Spiridonov, M. Toms, E. Vlasov, A. Zhokin

Moscow Institute of Physics and Technology, Moscow, Russia

A. Bylinkin ${ }^{36}$ 
National Research Nuclear University 'Moscow Engineering Physics Institute' (MEPhI), Moscow, Russia

R. Chistov ${ }^{39}$, M. Danilov ${ }^{39}$, S. Polikarpov

P.N. Lebedev Physical Institute, Moscow, Russia

V. Andreev, M. Azarkin ${ }^{36}$, I. Dremin ${ }^{36}$, M. Kirakosyan, A. Leonidov ${ }^{36}$, A. Terkulov

Skobeltsyn Institute of Nuclear Physics, Lomonosov Moscow State University, Moscow, Russia

A. Baskakov, A. Belyaev, E. Boos, V. Bunichev, M. Dubinin ${ }^{40}$, L. Dudko, A. Gribushin, V. Klyukhin, O. Kodolova, I. Lokhtin, I. Miagkov, S. Obraztsov, S. Petrushanko, V. Savrin, A. Snigirev

Novosibirsk State University (NSU), Novosibirsk, Russia

V. Blinov ${ }^{41}$, Y.Skovpen $^{41}$, D. Shtol ${ }^{41}$

State Research Center of Russian Federation, Institute for High Energy Physics, Protvino, Russia

I. Azhgirey, I. Bayshev, S. Bitioukov, D. Elumakhov, V. Kachanov, A. Kalinin, D. Konstantinov, V. Krychkine, V. Petrov, R. Ryutin, A. Sobol, S. Troshin, N. Tyurin, A. Uzunian,

A. Volkov

University of Belgrade, Faculty of Physics and Vinca Institute of Nuclear Sciences, Belgrade, Serbia

P. Adzic ${ }^{42}$, P. Cirkovic, D. Devetak, M. Dordevic, J. Milosevic, V. Rekovic

Centro de Investigaciones Energéticas Medioambientales y Tecnológicas (CIEMAT), Madrid, Spain

J. Alcaraz Maestre, M. Barrio Luna, E. Calvo, M. Cerrada, M. Chamizo Llatas, N. Colino, B. De La Cruz, A. Delgado Peris, A. Escalante Del Valle, C. Fernandez Bedoya, J.P. Fernández Ramos, J. Flix, M.C. Fouz, P. Garcia-Abia, O. Gonzalez Lopez, S. Goy Lopez, J.M. Hernandez, M.I. Josa, E. Navarro De Martino, A. Pérez-Calero Yzquierdo, J. Puerta Pelayo, A. Quintario Olmeda, I. Redondo, L. Romero, M.S. Soares

Universidad Autónoma de Madrid, Madrid, Spain

J.F. de Trocóniz, M. Missiroli, D. Moran

Universidad de Oviedo, Oviedo, Spain

J. Cuevas, J. Fernandez Menendez, I. Gonzalez Caballero, J.R. González Fernández, E. Palencia Cortezon, S. Sanchez Cruz, I. Suárez Andrés, J.M. Vizan Garcia

Instituto de Física de Cantabria (IFCA), CSIC-Universidad de Cantabria, Santander, Spain

I.J. Cabrillo, A. Calderon, J.R. Castiñeiras De Saa, E. Curras, M. Fernandez, J. GarciaFerrero, G. Gomez, A. Lopez Virto, J. Marco, C. Martinez Rivero, F. Matorras, J. Piedra Gomez, T. Rodrigo, A. Ruiz-Jimeno, L. Scodellaro, N. Trevisani, I. Vila, R. Vilar Cortabitarte 


\section{CERN, European Organization for Nuclear Research, Geneva, Switzerland}

D. Abbaneo, E. Auffray, G. Auzinger, M. Bachtis, P. Baillon, A.H. Ball, D. Barney, P. Bloch, A. Bocci, A. Bonato, C. Botta, T. Camporesi, R. Castello, M. Cepeda, G. Cerminara, Y. Chen, D. d'Enterria, A. Dabrowski, V. Daponte, A. David, M. De Gruttola, A. De Roeck, E. Di Marco ${ }^{43}$, M. Dobson, B. Dorney, T. du Pree, D. Duggan, M. Dünser, N. Dupont, A. Elliott-Peisert, P. Everaerts, S. Fartoukh, G. Franzoni, J. Fulcher, W. Funk, D. Gigi, K. Gill, M. Girone, F. Glege, D. Gulhan, S. Gundacker, M. Guthoff, J. Hammer, P. Harris, J. Hegeman, V. Innocente, P. Janot, J. Kieseler, H. Kirschenmann, V. Knünz, A. Kornmayer ${ }^{15}$, M.J. Kortelainen, K. Kousouris, M. Krammer ${ }^{1}$, C. Lange, P. Lecoq, C. Lourenço, M.T. Lucchini, L. Malgeri, M. Mannelli, A. Martelli, F. Meijers, J.A. Merlin, S. Mersi, E. Meschi, P. Milenovic ${ }^{44}$, F. Moortgat, S. Morovic, M. Mulders, H. Neugebauer, S. Orfanelli, L. Orsini, L. Pape, E. Perez, M. Peruzzi, A. Petrilli, G. Petrucciani, A. Pfeiffer, M. Pierini, A. Racz, T. Reis, G. Rolandi ${ }^{45}$, M. Rovere, H. Sakulin, J.B. Sauvan, C. Schäfer, C. Schwick, M. Seidel, A. Sharma, P. Silva, P. Sphicas ${ }^{46}$, J. Steggemann, M. Stoye, Y. Takahashi, M. Tosi, D. Treille, A. Triossi, A. Tsirou, V. Veckalns ${ }^{47}$, G.I. Veres ${ }^{20}$, M. Verweij, N. Wardle, H.K. Wöhri, A. Zagozdzinska ${ }^{34}$, W.D. Zeuner

\section{Paul Scherrer Institut, Villigen, Switzerland}

W. Bertl, K. Deiters, W. Erdmann, R. Horisberger, Q. Ingram, H.C. Kaestli, D. Kotlinski, U. Langenegger, T. Rohe

\section{Institute for Particle Physics, ETH Zurich, Zurich, Switzerland}

F. Bachmair, L. Bäni, L. Bianchini, B. Casal, G. Dissertori, M. Dittmar, M. Donegà, C. Grab, C. Heidegger, D. Hits, J. Hoss, G. Kasieczka, P. Lecomte ${ }^{\dagger}$, W. Lustermann, B. Mangano, M. Marionneau, P. Martinez Ruiz del Arbol, M. Masciovecchio, M.T. Meinhard, D. Meister, F. Micheli, P. Musella, F. Nessi-Tedaldi, F. Pandolfi, J. Pata, F. Pauss, G. Perrin, L. Perrozzi, M. Quittnat, M. Rossini, M. Schönenberger, A. Starodumov ${ }^{48}$, V.R. Tavolaro, K. Theofilatos, R. Wallny

\section{Universität Zürich, Zurich, Switzerland}

T.K. Aarrestad, C. Amsler ${ }^{49}$, L. Caminada, M.F. Canelli, A. De Cosa, C. Galloni, A. Hinzmann, T. Hreus, B. Kilminster, J. Ngadiuba, D. Pinna, G. Rauco, P. Robmann, D. Salerno, Y. Yang, A. Zucchetta

\section{National Central University, Chung-Li, Taiwan}

V. Candelise, T.H. Doan, Sh. Jain, R. Khurana, M. Konyushikhin, C.M. Kuo, W. Lin, Y.J. Lu, A. Pozdnyakov, S.S. Yu

National Taiwan University (NTU), Taipei, Taiwan

Arun Kumar, P. Chang, Y.H. Chang, Y. Chao, K.F. Chen, P.H. Chen, F. Fiori, W.-S. Hou, Y. Hsiung, Y.F. Liu, R.-S. Lu, M. Miñano Moya, E. Paganis, A. Psallidas, J.f. Tsai

Chulalongkorn University, Faculty of Science, Department of Physics, Bangkok, Thailand

B. Asavapibhop, G. Singh, N. Srimanobhas, N. Suwonjandee 
Cukurova University - Physics Department, Science and Art Faculty

A. Adiguzel, S. Damarseckin, Z.S. Demiroglu, C. Dozen, E. Eskut, S. Girgis, G. Gokbulut, Y. Guler, I. Hos ${ }^{50}$, E.E. Kangal ${ }^{51}$, O. Kara, A. Kayis Topaksu, U. Kiminsu, M. Oglakci, G. Onengut ${ }^{52}$, K. Ozdemir ${ }^{53}$, S. Ozturk ${ }^{54}$, A. Polatoz, B. Tali ${ }^{55}$, S. Turkcapar, I.S. Zorbakir, C. Zorbilmez

Middle East Technical University, Physics Department, Ankara, Turkey

B. Bilin, S. Bilmis, B. Isildak ${ }^{56}$, G. Karapinar ${ }^{57}$, M. Yalvac, M. Zeyrek

Bogazici University, Istanbul, Turkey

E. Gülmez, M. Kaya ${ }^{58}$, O. Kaya ${ }^{59}$, E.A. Yetkin ${ }^{60}$, T. Yetkin ${ }^{61}$

Istanbul Technical University, Istanbul, Turkey

A. Cakir, K. Cankocak, S. Sen ${ }^{62}$

Institute for Scintillation Materials of National Academy of Science of Ukraine, Kharkov, Ukraine

B. Grynyov

National Scientific Center, Kharkov Institute of Physics and Technology, Kharkov, Ukraine

L. Levchuk, P. Sorokin

University of Bristol, Bristol, United Kingdom

R. Aggleton, F. Ball, L. Beck, J.J. Brooke, D. Burns, E. Clement, D. Cussans, H. Flacher,

J. Goldstein, M. Grimes, G.P. Heath, H.F. Heath, J. Jacob, L. Kreczko, C. Lucas, D.M. Newbold ${ }^{63}$, S. Paramesvaran, A. Poll, T. Sakuma, S. Seif El Nasr-storey, D. Smith, V.J. Smith

Rutherford Appleton Laboratory, Didcot, United Kingdom

K.W. Bell, A. Belyaev ${ }^{64}$, C. Brew, R.M. Brown, L. Calligaris, D. Cieri, D.J.A. Cockerill, J.A. Coughlan, K. Harder, S. Harper, E. Olaiya, D. Petyt, C.H. Shepherd-Themistocleous, A. Thea, I.R. Tomalin, T. Williams

Imperial College, London, United Kingdom

M. Baber, R. Bainbridge, O. Buchmuller, A. Bundock, D. Burton, S. Casasso, M. Citron,

D. Colling, L. Corpe, P. Dauncey, G. Davies, A. De Wit, M. Della Negra, R. Di Maria,

P. Dunne, A. Elwood, D. Futyan, Y. Haddad, G. Hall, G. Iles, T. James, R. Lane,

C. Laner, R. Lucas ${ }^{63}$, L. Lyons, A.-M. Magnan, S. Malik, L. Mastrolorenzo, J. Nash,

A. Nikitenko ${ }^{48}$, J. Pela, B. Penning, M. Pesaresi, D.M. Raymond, A. Richards, A. Rose,

C. Seez, S. Summers, A. Tapper, K. Uchida, M. Vazquez Acosta ${ }^{65}$, T. Virdee ${ }^{15}$, J. Wright, S.C. Zenz

Brunel University, Uxbridge, United Kingdom

J.E. Cole, P.R. Hobson, A. Khan, P. Kyberd, D. Leslie, I.D. Reid, P. Symonds, L. Teodorescu, M. Turner

Baylor University, Waco, USA

A. Borzou, K. Call, J. Dittmann, K. Hatakeyama, H. Liu, N. Pastika 
The University of Alabama, Tuscaloosa, USA

S.I. Cooper, C. Henderson, P. Rumerio, C. West

\section{Boston University, Boston, USA}

D. Arcaro, A. Avetisyan, T. Bose, D. Gastler, D. Rankin, C. Richardson, J. Rohlf, L. Sulak, D. Zou

\section{Brown University, Providence, USA}

G. Benelli, D. Cutts, A. Garabedian, J. Hakala, U. Heintz, J.M. Hogan, O. Jesus, K.H.M. Kwok, E. Laird, G. Landsberg, Z. Mao, M. Narain, S. Piperov, S. Sagir, E. Spencer, R. Syarif

\section{University of California, Davis, Davis, USA}

R. Breedon, D. Burns, M. Calderon De La Barca Sanchez, S. Chauhan, M. Chertok, J. Conway, R. Conway, P.T. Cox, R. Erbacher, C. Flores, G. Funk, M. Gardner, W. Ko, R. Lander, C. Mclean, M. Mulhearn, D. Pellett, J. Pilot, S. Shalhout, J. Smith, M. Squires, D. Stolp, M. Tripathi

\section{University of California, Los Angeles, USA}

C. Bravo, R. Cousins, A. Dasgupta, A. Florent, J. Hauser, M. Ignatenko, N. Mccoll, D. Saltzberg, C. Schnaible, V. Valuev, M. Weber

\section{University of California, Riverside, Riverside, USA}

E. Bouvier, K. Burt, R. Clare, J. Ellison, J.W. Gary, S.M.A. Ghiasi Shirazi, G. Hanson, J. Heilman, P. Jandir, E. Kennedy, F. Lacroix, O.R. Long, M. Olmedo Negrete, M.I. Paneva, A. Shrinivas, W. Si, H. Wei, S. Wimpenny, B. R. Yates

\section{University of California, San Diego, La Jolla, USA}

J.G. Branson, G.B. Cerati, S. Cittolin, M. Derdzinski, R. Gerosa, A. Holzner, D. Klein, V. Krutelyov, J. Letts, I. Macneill, D. Olivito, S. Padhi, M. Pieri, M. Sani, V. Sharma, S. Simon, M. Tadel, A. Vartak, S. Wasserbaech ${ }^{66}$, C. Welke, J. Wood, F. Würthwein, A. Yagil, G. Zevi Della Porta

University of California, Santa Barbara - Department of Physics, Santa Barbara, USA

N. Amin, R. Bhandari, J. Bradmiller-Feld, C. Campagnari, A. Dishaw, V. Dutta, M. Franco Sevilla, C. George, F. Golf, L. Gouskos, J. Gran, R. Heller, J. Incandela, S.D. Mullin, A. Ovcharova, H. Qu, J. Richman, D. Stuart, I. Suarez, J. Yoo

\section{California Institute of Technology, Pasadena, USA}

D. Anderson, J. Bendavid, A. Bornheim, J. Bunn, J. Duarte, J.M. Lawhorn, A. Mott, H.B. Newman, C. Pena, M. Spiropulu, J.R. Vlimant, S. Xie, R.Y. Zhu

\section{Carnegie Mellon University, Pittsburgh, USA}

M.B. Andrews, T. Ferguson, M. Paulini, J. Russ, M. Sun, H. Vogel, I. Vorobiev, M. Weinberg 


\section{University of Colorado Boulder, Boulder, USA}

J.P. Cumalat, W.T. Ford, F. Jensen, A. Johnson, M. Krohn, T. Mulholland, K. Stenson, S.R. Wagner

\section{Cornell University, Ithaca, USA}

J. Alexander, J. Chaves, J. Chu, S. Dittmer, K. Mcdermott, N. Mirman, G. Nicolas Kaufman, J.R. Patterson, A. Rinkevicius, A. Ryd, L. Skinnari, L. Soffi, S.M. Tan, Z. Tao, J. Thom, J. Tucker, P. Wittich, M. Zientek

\section{Fairfield University, Fairfield, USA}

D. Winn

\section{Fermi National Accelerator Laboratory, Batavia, USA}

S. Abdullin, M. Albrow, G. Apollinari, A. Apresyan, S. Banerjee, L.A.T. Bauerdick, A. Beretvas, J. Berryhill, P.C. Bhat, G. Bolla, K. Burkett, J.N. Butler, H.W.K. Cheung, F. Chlebana, S. Cihangir ${ }^{\dagger}$, M. Cremonesi, V.D. Elvira, I. Fisk, J. Freeman, E. Gottschalk, L. Gray, D. Green, S. Grünendahl, O. Gutsche, D. Hare, R.M. Harris, S. Hasegawa, J. Hirschauer, Z. Hu, B. Jayatilaka, S. Jindariani, M. Johnson, U. Joshi, B. Klima, B. Kreis, S. Lammel, J. Linacre, D. Lincoln, R. Lipton, M. Liu, T. Liu, R. Lopes De Sá, J. Lykken, K. Maeshima, N. Magini, J.M. Marraffino, S. Maruyama, D. Mason, P. McBride, P. Merkel, S. Mrenna, S. Nahn, V. O'Dell, K. Pedro, O. Prokofyev, G. Rakness, L. Ristori, E. SextonKennedy, A. Soha, W.J. Spalding, L. Spiegel, S. Stoynev, J. Strait, N. Strobbe, L. Taylor, S. Tkaczyk, N.V. Tran, L. Uplegger, E.W. Vaandering, C. Vernieri, M. Verzocchi, R. Vidal, M. Wang, H.A. Weber, A. Whitbeck, Y. Wu

\section{University of Florida, Gainesville, USA}

D. Acosta, P. Avery, P. Bortignon, D. Bourilkov, A. Brinkerhoff, A. Carnes, M. Carver, D. Curry, S. Das, R.D. Field, I.K. Furic, J. Konigsberg, A. Korytov, J.F. Low, P. Ma, K. Matchev, H. Mei, G. Mitselmakher, D. Rank, L. Shchutska, D. Sperka, L. Thomas, J. Wang, S. Wang, J. Yelton

\section{Florida International University, Miami, USA}

S. Linn, P. Markowitz, G. Martinez, J.L. Rodriguez

\section{Florida State University, Tallahassee, USA}

A. Ackert, T. Adams, A. Askew, S. Bein, S. Hagopian, V. Hagopian, K.F. Johnson, H. Prosper, A. Santra, R. Yohay

\section{Florida Institute of Technology, Melbourne, USA}

M.M. Baarmand, V. Bhopatkar, S. Colafranceschi, M. Hohlmann, D. Noonan, T. Roy, F. Yumiceva

\section{University of Illinois at Chicago (UIC), Chicago, USA}

M.R. Adams, L. Apanasevich, D. Berry, R.R. Betts, I. Bucinskaite, R. Cavanaugh, O. Evdokimov, L. Gauthier, C.E. Gerber, D.J. Hofman, K. Jung, I.D. Sandoval Gonzalez, N. Varelas, H. Wang, Z. Wu, M. Zakaria, J. Zhang 
The University of Iowa, Iowa City, USA

B. Bilki ${ }^{67}$, W. Clarida, K. Dilsiz, S. Durgut, R.P. Gandrajula, M. Haytmyradov, V. Khristenko, J.-P. Merlo, H. Mermerkaya ${ }^{68}$, A. Mestvirishvili, A. Moeller, J. Nachtman, H. Ogul, Y. Onel, F. Ozok ${ }^{69}$, A. Penzo, C. Snyder, E. Tiras, J. Wetzel, K. Yi

Johns Hopkins University, Baltimore, USA

I. Anderson, B. Blumenfeld, A. Cocoros, N. Eminizer, D. Fehling, L. Feng, A.V. Gritsan, P. Maksimovic, C. Martin, M. Osherson, J. Roskes, U. Sarica, M. Swartz, M. Xiao, Y. Xin, C. You

\section{The University of Kansas, Lawrence, USA}

A. Al-bataineh, P. Baringer, A. Bean, S. Boren, J. Bowen, J. Castle, L. Forthomme, R.P. Kenny III, S. Khalil, A. Kropivnitskaya, D. Majumder, W. Mcbrayer, M. Murray, S. Sanders, R. Stringer, J.D. Tapia Takaki, Q. Wang, G. Wilson

\section{Kansas State University, Manhattan, USA}

A. Ivanov, K. Kaadze, Y. Maravin, A. Mohammadi, L.K. Saini, N. Skhirtladze, S. Toda

\section{Lawrence Livermore National Laboratory, Livermore, USA}

F. Rebassoo, D. Wright

\section{University of Maryland, College Park, USA}

C. Anelli, A. Baden, O. Baron, A. Belloni, B. Calvert, S.C. Eno, C. Ferraioli, J.A. Gomez, N.J. Hadley, S. Jabeen, R.G. Kellogg, T. Kolberg, J. Kunkle, Y. Lu, A.C. Mignerey, F. Ricci-Tam, Y.H. Shin, A. Skuja, M.B. Tonjes, S.C. Tonwar

\section{Massachusetts Institute of Technology, Cambridge, USA}

D. Abercrombie, B. Allen, A. Apyan, V. Azzolini, R. Barbieri, A. Baty, R. Bi, K. Bierwagen, S. Brandt, W. Busza, I.A. Cali, M. D'Alfonso, Z. Demiragli, L. Di Matteo, G. Gomez Ceballos, M. Goncharov, D. Hsu, Y. Iiyama, G.M. Innocenti, M. Klute, D. Kovalskyi, K. Krajczar, Y.S. Lai, Y.-J. Lee, A. Levin, P.D. Luckey, B. Maier, A.C. Marini, C. Mcginn, C. Mironov, S. Narayanan, X. Niu, C. Paus, C. Roland, G. Roland, J. Salfeld-Nebgen, G.S.F. Stephans, K. Tatar, M. Varma, D. Velicanu, J. Veverka, J. Wang, T.W. Wang, B. Wyslouch, M. Yang

\section{University of Minnesota, Minneapolis, USA}

A.C. Benvenuti, R.M. Chatterjee, A. Evans, A. Finkel, A. Gude, P. Hansen, S. Kalafut, S.C. Kao, Y. Kubota, Z. Lesko, J. Mans, S. Nourbakhsh, N. Ruckstuhl, R. Rusack, N. Tambe, J. Turkewitz

\section{University of Mississippi, Oxford, USA}

J.G. Acosta, S. Oliveros

\section{University of Nebraska-Lincoln, Lincoln, USA}

E. Avdeeva, R. Bartek ${ }^{70}$, K. Bloom, D.R. Claes, A. Dominguez ${ }^{70}$, C. Fangmeier, R. Gonzalez Suarez, R. Kamalieddin, I. Kravchenko, A. Malta Rodrigues, F. Meier, J. Monroy, J.E. Siado, G.R. Snow, B. Stieger 
State University of New York at Buffalo, Buffalo, USA

M. Alyari, J. Dolen, A. Godshalk, C. Harrington, I. Iashvili, J. Kaisen, A. Kharchilava, A. Parker, S. Rappoccio, B. Roozbahani

Northeastern University, Boston, USA

G. Alverson, E. Barberis, A. Hortiangtham, A. Massironi, D.M. Morse, D. Nash, T. Orimoto, R. Teixeira De Lima, D. Trocino, R.-J. Wang, D. Wood

Northwestern University, Evanston, USA

S. Bhattacharya, O. Charaf, K.A. Hahn, A. Kumar, N. Mucia, N. Odell, B. Pollack, M.H. Schmitt, K. Sung, M. Trovato, M. Velasco

\section{University of Notre Dame, Notre Dame, USA}

N. Dev, M. Hildreth, K. Hurtado Anampa, C. Jessop, D.J. Karmgard, N. Kellams, K. Lannon, N. Marinelli, F. Meng, C. Mueller, Y. Musienko ${ }^{35}$, M. Planer, A. Reinsvold, R. Ruchti, G. Smith, S. Taroni, M. Wayne, M. Wolf, A. Woodard

The Ohio State University, Columbus, USA

J. Alimena, L. Antonelli, B. Bylsma, L.S. Durkin, S. Flowers, B. Francis, A. Hart, C. Hill, R. Hughes, W. Ji, B. Liu, W. Luo, D. Puigh, B.L. Winer, H.W. Wulsin

Princeton University, Princeton, USA

S. Cooperstein, O. Driga, P. Elmer, J. Hardenbrook, P. Hebda, D. Lange, J. Luo, D. Marlow, T. Medvedeva, K. Mei, J. Olsen, C. Palmer, P. Piroué, D. Stickland, A. Svyatkovskiy, C. Tully

University of Puerto Rico, Mayaguez, USA

S. Malik

Purdue University, West Lafayette, USA

A. Barker, V.E. Barnes, S. Folgueras, L. Gutay, M.K. Jha, M. Jones, A.W. Jung, A. Khatiwada, D.H. Miller, N. Neumeister, J.F. Schulte, X. Shi, J. Sun, F. Wang, W. Xie Purdue University Calumet, Hammond, USA

N. Parashar, J. Stupak

\section{Rice University, Houston, USA}

A. Adair, B. Akgun, Z. Chen, K.M. Ecklund, F.J.M. Geurts, M. Guilbaud, W. Li, B. Michlin, M. Northup, B.P. Padley, J. Roberts, J. Rorie, Z. Tu, J. Zabel

University of Rochester, Rochester, USA

B. Betchart, A. Bodek, P. de Barbaro, R. Demina, Y.t. Duh, T. Ferbel, M. Galanti, A. Garcia-Bellido, J. Han, O. Hindrichs, A. Khukhunaishvili, K.H. Lo, P. Tan, M. Verzetti

\section{Rutgers, The State University of New Jersey, Piscataway, USA}

A. Agapitos, J.P. Chou, Y. Gershtein, T.A. Gómez Espinosa, E. Halkiadakis, M. Heindl, E. Hughes, S. Kaplan, R. Kunnawalkam Elayavalli, S. Kyriacou, A. Lath, K. Nash, H. Saka, S. Salur, S. Schnetzer, D. Sheffield, S. Somalwar, R. Stone, S. Thomas, P. Thomassen, M. Walker 


\section{University of Tennessee, Knoxville, USA}

A.G. Delannoy, M. Foerster, J. Heideman, G. Riley, K. Rose, S. Spanier, K. Thapa

\section{Texas A\&M University, College Station, USA}

O. Bouhali ${ }^{71}$, A. Celik, M. Dalchenko, M. De Mattia, A. Delgado, S. Dildick, R. Eusebi, J. Gilmore, T. Huang, E. Juska, T. Kamon ${ }^{72}$, R. Mueller, Y. Pakhotin, R. Patel, A. Perloff, L. Perniè, D. Rathjens, A. Safonov, A. Tatarinov, K.A. Ulmer

\section{Texas Tech University, Lubbock, USA}

N. Akchurin, C. Cowden, J. Damgov, F. De Guio, C. Dragoiu, P.R. Dudero, J. Faulkner, E. Gurpinar, S. Kunori, K. Lamichhane, S.W. Lee, T. Libeiro, T. Peltola, S. Undleeb, I. Volobouev, Z. Wang

\section{Vanderbilt University, Nashville, USA}

S. Greene, A. Gurrola, R. Janjam, W. Johns, C. Maguire, A. Melo, H. Ni, P. Sheldon, S. Tuo, J. Velkovska, Q. Xu

\section{University of Virginia, Charlottesville, USA}

M.W. Arenton, P. Barria, B. Cox, J. Goodell, R. Hirosky, A. Ledovskoy, H. Li, C. Neu, T. Sinthuprasith, X. Sun, Y. Wang, E. Wolfe, F. Xia

\section{Wayne State University, Detroit, USA}

C. Clarke, R. Harr, P.E. Karchin, J. Sturdy

\section{University of Wisconsin - Madison, Madison, WI, USA}

D.A. Belknap, J. Buchanan, C. Caillol, S. Dasu, L. Dodd, S. Duric, B. Gomber, M. Grothe, M. Herndon, A. Hervé, P. Klabbers, A. Lanaro, A. Levine, K. Long, R. Loveless, I. Ojalvo, T. Perry, G.A. Pierro, G. Polese, T. Ruggles, A. Savin, N. Smith, W.H. Smith, D. Taylor, N. Woods

\section{$\dagger$ : Deceased}

1: Also at Vienna University of Technology, Vienna, Austria

2: Also at State Key Laboratory of Nuclear Physics and Technology, Peking University, Beijing, China

3: Also at Institut Pluridisciplinaire Hubert Curien (IPHC), Université de Strasbourg, CNRS/IN2P3, Strasbourg, France

4: Also at Universidade Estadual de Campinas, Campinas, Brazil

5: Also at Universidade Federal de Pelotas, Pelotas, Brazil

6: Also at Université Libre de Bruxelles, Bruxelles, Belgium

7: Also at Deutsches Elektronen-Synchrotron, Hamburg, Germany

8: Also at Joint Institute for Nuclear Research, Dubna, Russia

9: Now at Cairo University, Cairo, Egypt

10: Also at Fayoum University, El-Fayoum, Egypt

11: Now at British University in Egypt, Cairo, Egypt

12: Now at Ain Shams University, Cairo, Egypt

13: Also at Université de Haute Alsace, Mulhouse, France 
14: Also at Skobeltsyn Institute of Nuclear Physics, Lomonosov Moscow State University, Moscow, Russia

15: Also at CERN, European Organization for Nuclear Research, Geneva, Switzerland

16: Also at RWTH Aachen University, III. Physikalisches Institut A, Aachen, Germany

17: Also at University of Hamburg, Hamburg, Germany

18: Also at Brandenburg University of Technology, Cottbus, Germany

19: Also at Institute of Nuclear Research ATOMKI, Debrecen, Hungary

20: Also at MTA-ELTE Lendület CMS Particle and Nuclear Physics Group, Eötvös Loránd University, Budapest, Hungary

21: Also at Institute of Physics, University of Debrecen, Debrecen, Hungary

22: Also at Indian Institute of Science Education and Research, Bhopal, India

23: Also at Institute of Physics, Bhubaneswar, India

24: Also at University of Visva-Bharati, Santiniketan, India

25: Also at University of Ruhuna, Matara, Sri Lanka

26: Also at Isfahan University of Technology, Isfahan, Iran

27: Also at Yazd University, Yazd, Iran

28: Also at Plasma Physics Research Center, Science and Research Branch, Islamic Azad University, Tehran, Iran

29: Also at Università degli Studi di Siena, Siena, Italy

30: Also at Purdue University, West Lafayette, USA

31: Also at International Islamic University of Malaysia, Kuala Lumpur, Malaysia

32: Also at Malaysian Nuclear Agency, MOSTI, Kajang, Malaysia

33: Also at Consejo Nacional de Ciencia y Tecnología, Mexico city, Mexico

34: Also at Warsaw University of Technology, Institute of Electronic Systems, Warsaw, Poland

35: Also at Institute for Nuclear Research, Moscow, Russia

36: Now at National Research Nuclear University 'Moscow Engineering Physics Institute' (MEPhI), Moscow, Russia

37: Also at St. Petersburg State Polytechnical University, St. Petersburg, Russia

38: Also at University of Florida, Gainesville, USA

39: Also at P.N. Lebedev Physical Institute, Moscow, Russia

40: Also at California Institute of Technology, Pasadena, USA

41: Also at Budker Institute of Nuclear Physics, Novosibirsk, Russia

42: Also at Faculty of Physics, University of Belgrade, Belgrade, Serbia

43: Also at INFN Sezione di Roma; Università di Roma, Roma, Italy

44: Also at University of Belgrade, Faculty of Physics and Vinca Institute of Nuclear Sciences, Belgrade, Serbia

45: Also at Scuola Normale e Sezione dell'INFN, Pisa, Italy

46: Also at National and Kapodistrian University of Athens, Athens, Greece

47: Also at Riga Technical University, Riga, Latvia

48: Also at Institute for Theoretical and Experimental Physics, Moscow, Russia

49: Also at Albert Einstein Center for Fundamental Physics, Bern, Switzerland

50: Also at Istanbul Aydin University, Istanbul, Turkey

51: Also at Mersin University, Mersin, Turkey

52: Also at Cag University, Mersin, Turkey

53: Also at Piri Reis University, Istanbul, Turkey

54: Also at Gaziosmanpasa University, Tokat, Turkey

55: Also at Adiyaman University, Adiyaman, Turkey

56: Also at Ozyegin University, Istanbul, Turkey 
57: Also at Izmir Institute of Technology, Izmir, Turkey

58: Also at Marmara University, Istanbul, Turkey

59: Also at Kafkas University, Kars, Turkey

60: Also at Istanbul Bilgi University, Istanbul, Turkey

61: Also at Yildiz Technical University, Istanbul, Turkey

62: Also at Hacettepe University, Ankara, Turkey

63: Also at Rutherford Appleton Laboratory, Didcot, United Kingdom

64: Also at School of Physics and Astronomy, University of Southampton, Southampton, United Kingdom

65: Also at Instituto de Astrofísica de Canarias, La Laguna, Spain

66: Also at Utah Valley University, Orem, USA

67: Also at Argonne National Laboratory, Argonne, USA

68: Also at Erzincan University, Erzincan, Turkey

69: Also at Mimar Sinan University, Istanbul, Istanbul, Turkey

70: Now at The Catholic University of America, Washington, USA

71: Also at Texas A\&M University at Qatar, Doha, Qatar

72: Also at Kyungpook National University, Daegu, Korea 